\title{
Deformation of metallic glasses: Recent developments in theory, simulations, and experiments.
}

\author{
Todd C. Hufnagel ${ }^{a, *}$, Christopher A. Schuh ${ }^{\mathrm{b}, * *}$, Michael L. Falk ${ }^{\mathrm{a}, * *}$ \\ ${ }^{a}$ Department of Materials Science and Engineering, Johns Hopkins University, Baltimore, \\ MD USA \\ ${ }^{b}$ Department of Materials Science and Engineering, Massachusetts Institute of Technology, \\ Cambridge, MA USA
}

\begin{abstract}
We review recent research into elastic and plastic deformation of metallic glasses, with an emphasis on making connections between developments in theory and simulation (largely from the physics community) and experimental results (largely from the metallurgy community). Topics covered include strain measurement via scattering techniques, non-affine atomic displacements during elastic deformation, shear transformations, constitutive equations, shear bands, and strain hardening. Where possible we connect the observed behavior and properties to the structure of the glass on the atomic- and nano- scales.

Keywords: Metallic glass, amorphous alloy, disordered material, elastic deformation, plastic deformation.
\end{abstract}

\section{Introduction}

Over the more than fifty years since their discovery [1] there has been extensive research into the structure and properties of metallic glasses. The earliest metallic glasses required rapid solidification and could therefore be produced 5 only in limited forms. The development of bulk glass-forming alloys 2 -4 not only provided samples suitable for traditional mechanical testing techniques but

\footnotetext{
${ }^{*}$ Principal corresponding author

${ }^{* *}$ Corresponding author

Email addresses: hufnagel@jhu.edu (Todd C. Hufnagel), schuh@mit.edu (Christopher A. Schuh), mfalk@jhu.edu (Michael L. Falk)

Preprint submitted to Acta Materialia

January 18, 2016
}

(C) 2016. This manuscript version is made available under the Elsevier user license http://www.elsevier.com/open-access/userlicense/1.0/ 
also raised the possibility that amorphous alloys might be employed in structural applications.

In 2007, two of us were co-authors on a review article summarizing the state of understanding of mechanical behavior of metallic glasses and proposing directions for future research [5]. Since then interest in the mechanical behavior of metallic glasses has remained intense and an updated review seems timely. In this article we review several areas where significant progress has been made and develop a few new ideas, particularly with regard to insights from theoretical and computational investigations of mechanical response of amorphous alloys (and disordered materials more generally). For a more comprehensive discussion we refer the reader to the earlier review [5] or to several other reviews of mechanical behavior of metallic glasses that have been published since 2007 [6-11].

The central paradigm of materials science states that the structure, proper20 ties, and processing of materials are interrelated. The periodicity of crystalline materials facilitates connections between their mechanical response and their atomic-scale structure, both experimentally (for example, by making dislocations visible in transmission electron microscopy) and theoretically. Such connections are much more challenging in metallic glasses, which lack long-range order and therefore are not as amenable to detailed characterization by traditional diffraction-based techniques. Nevertheless, experimental investigations using nanoscale probes now complement a rich set of computer simulations and theoretical analyses [12, 13] to provide an emerging consensus of the phenomenology of the structural origin of mechanical response in these materials.

Connections between structure and properties, therefore, provide a unifying theme for the topics discussed in this review. After a brief review of our current understanding of the structure of metallic glasses, we begin our discussion of mechanical behavior at the atomic- and nano-scale by considering the use of scattering techniques for measuring elastic strain in metallic glasses, and the 35 implications and limitations of this new technique. This leads into a discussion of non-affine atomic displacements during elastic deformation and the connection of these features to plastic deformation through shear transformations. We next 
consider how the structure of metallic glasses evolves under load and recent attempts to capture this evolution in the form of constitutive relations that could be useful for modeling of mechanical behavior. Moving to larger length scales, we examine localized plastic flow in shear bands with an emphasis on recent studies of their structure and dynamics. This introduces the topic of strain hardening in metallic glasses, through two recently-reported mechanisms: Exhaustion hardening and inherent hardening by relaxation. As in our original

45 review [5], the last section points out important and promising directions for future research.

\section{Structure of metallic glasses}

The salient feature of the structure of metallic glasses is that they are disordered - a statement that is not as much of a truism as it may appear. The possible structural states of matter occupy a continuum from the perfect regularity and homogeneity of defect-free crystals to the utter disorder of ideal gases. Liquids and glasses are undoubtedly among the more disordered forms of condensed matter, but exactly how much structural order exists, what form it takes, and the length scale over which it extends are difficult questions that 55 are the subject of ongoing research. The literature on this subject is extensive, but fortunately a comprehensive review of the structure of metallic glasses has recently been provided by Cheng and Ma $[$, along with a more recent brief review by $\mathrm{Ma}$ [14. In this section we highlight several key results that are important for understanding what follows; the interested reader is referred to those papers for a more complete discussion.

To begin at the shortest length scales, the high density and non-directional bonding of metallic glasses imply a high degree of short-range order based on close-packing of atoms. The basic packing unit is a tetrahedral cluster of four atoms; by allowing each atom to participate in several such clusters it is possible to achieve a high packing density, but because it is impossible to fill space with tetrahedra some degree of geometrical frustration is required (Fig. 1). Different 
Figure 1: Space-filling packing of regular tetrahedra is not possible, as illustrated here for the case of five tetrahedra sharing a common edge. (Figure based on an original in Ref. [16.)

glasses have different structural motifs, or preferred ways of packing tetrahedra (and accommodating the inevitable geometrical frustration). Chen and Ma refer to these different tetrahedral packings as "Z clusters" [8, 14] (Fig. 2). One much-discussed $\mathrm{Z}$ cluster consists of twelve atoms arranged at the vertices of a icosahedron around a central atom; in such a cluster the central atom plus any three mutually adjacent surface atoms form a tetrahedron (usually distorted). Although "five-fold" bonds - clusters where two adjacent atoms have five neighbors in common - are frequently observed, they should not be taken as indicative of icosahedral clustering which is, in fact, not very common. Rather, the presence of the five-fold bonds reflects dense polytetrahedral packing with a minimum of rotational defects [14, 15].

An example of the connection of atomic-scale structure to mechanical behavior is provided by atomistic simulations of $\mathrm{Cu}-\mathrm{Zr}$ alloys. The fraction of $\mathrm{Cu}$-centered icosahedral clusters increases dramatically as the liquid is cooled towards the glass transition, which is correlated with slowing dynamics of the system [15, 18, 19]. Adding $\mathrm{Al}$ to this simulated alloy enhances the development of icosahedral clusters, further slowing the dynamics 20. The influence of short-range order extends to the glassy state where locally greater icosahedral order correlates with higher stiffness and resistance to yield. Regions of the 
(a)

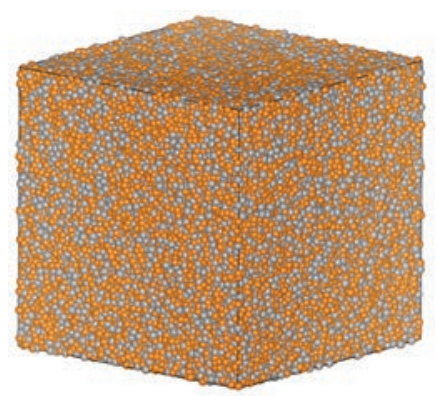

(c)

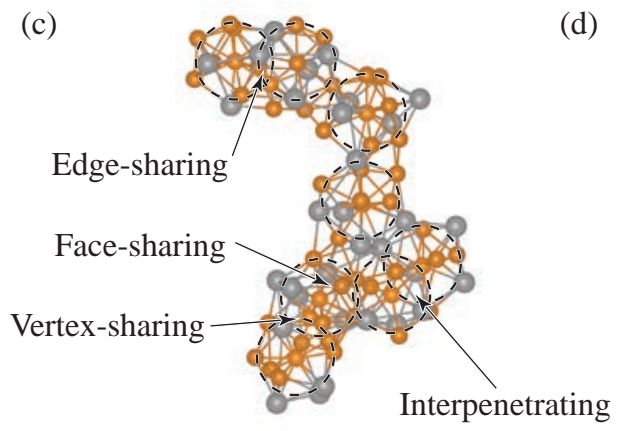

(b)

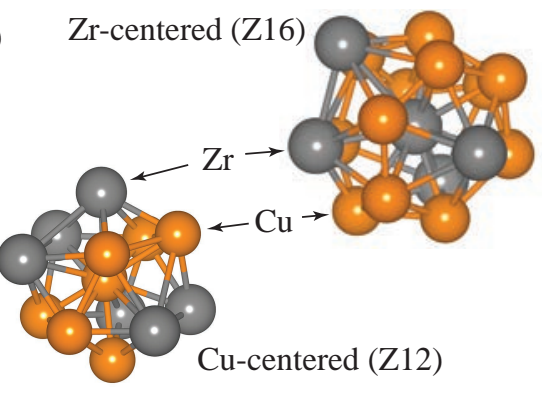

(d)

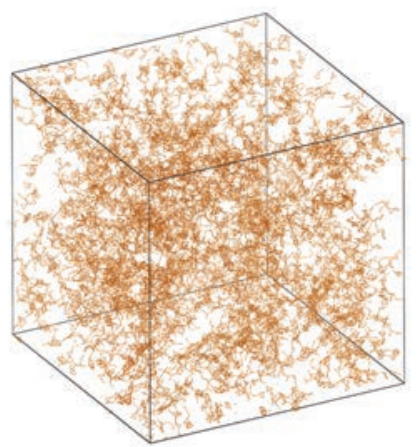

Figure 2: (a) Atomic configuration of a $\mathrm{Cu}_{64} \mathrm{Zr}_{36}$ metallic glass from molecular dynamics simulation 17] with 128,000 atoms and a box size of $12.74 \mathrm{~nm}$. Gold spheres represent $\mathrm{Zr}$ atoms and grey, Cu. (b) Representative atomic clusters centered on $\mathrm{Cu}$ and $\mathrm{Zr}$. The preferred cluster around copper is a full icosahedron with 12 nearest neighbors (Z12) while the larger $\mathrm{Zr}$ atoms can accommodate clusters with 16 near-neighbors (Z16). (c) A supercluster of 79 atoms, with eight interconnected $\mathrm{Cu}$-centered full icosahedra indicated by dashed circles. Clusters can interconnect by sharing vertices, edges, or faces, or they can be interpenetrating. (d) Network of interconnected icosahedra indicated by line segments connecting the $\mathrm{Cu}$ atoms at the center of each cluster. (Images provided by Jun Ding and En Ma.) 
structure without this favorable short-range order, which have been called "geometrically unfavorable motifs" (GUMs) [14], have lower stiffness and a greater tendency to undergo shear transformations [21, 22] as will be discussed in more detail below.

This last observation points out the importance of structural heterogeneity for understanding mechanical behavior of metallic glasses. It is reasonable to suspect that this heterogeneity may reflect the structure of the supercooled liquid from which the glass forms. Because of intense interest in the fundamental nature of the glass transition itself, the structure and dynamics of supercooled liquids near the glass transition have been the subject of extensive research [23] and there is strong evidence of increasing inhomogeneity upon cooling towards the glass transition [24, 25].

Beyond the nearest-neighbor interactions some kind of so-called "mediumrange" order is required to achieve the high densities observed for metallic glasses. The most developed theory of medium-range order in metallic glasses is the efficient cluster packing model of Miracle and coworkers [26, 27. This model begins with short-range order clusters similar to those discussed above (but centered on solute atoms) and then posits that these clusters are themselves arranged to produce efficient overall packing, for instance into fcc arrays. The structural order is presumed to extend over only a few cluster diameters. The densities predicted on the basis of this model are in reasonable agreement with experiment, and the model has some success in reproducing experimental measurements of the separations of solute atom pairs [27]. As with the nearest-neighbor interactions, it is reasonable to suppose that the structure is heterogeneous on these larger length scales.

\section{Elastic deformation}

To a metallurgist, elastic deformation of crystals is among the simplest mechanical behaviors to understand. At small strains $(<0.2 \%)$ deformation is fully reversible and follows Hooke's law (although non-linear and anelastic behavior 
(a)

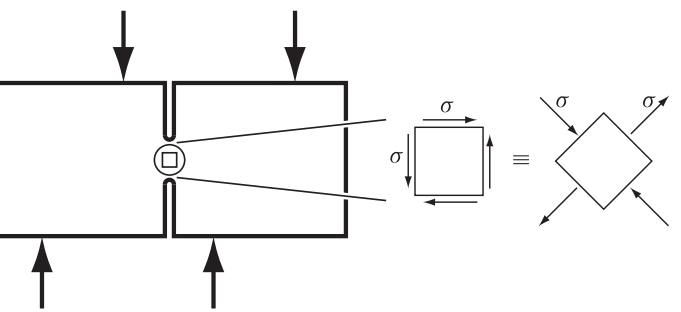

(b)

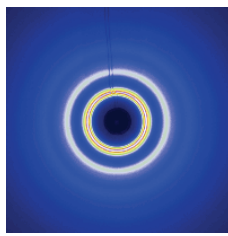

Unloaded

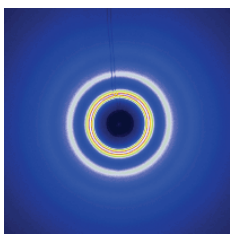

Under load

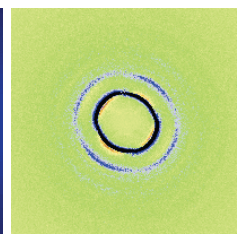

Difference

Figure 3: Elastic strain in a metallic glass can be measured from the distortion of the rings of scattered radiation. (a) Schematic of an asymmetric four-point bend specimen (left). Under load a state of nominally pure shear stress $\sigma$ is produced in the test region, which is equivalent to tensile and compressive normal stresses of the same magnitude in a coordinate system rotated by $45^{\circ}$ to the original (right). (b) Corresponding scattering measurements from a $\mathrm{Zr}$ based metallic glass. In the strain-free state (left) the scattering rings are circular. Loading to near the nominal yield stress in shear (center) distorts the rings into ellipses. The distortion is too small to see at this scale, but is clearly shown by taking the difference of the loaded and unloaded scattering patterns (right).

where $d_{\circ}$ and $q_{\circ}$ are the average interatomic spacing and peak position in the strain-free state, and $d_{\sigma}$ and $q_{\sigma}$ are the corresponding values under a macroscopic stress $\sigma$. To keep our notation simple we have written $\epsilon$ as a scalar although in reality it is a component of the strain tensor, and the values of $d_{\sigma}$ and $q_{\sigma}$ depend on the loading condition (Fig. 3] 32.

Although Eqn. 1 has been widely used, some caution is necessary. Implicit in the treatment leading to Eqn. 1 is the assumption that the structure of the material is not strongly perturbed by the strain state [33. This is probably a reasonable assumption for small elastic strains (although as noted in Sec. 4 below there is growing evidence of irreversible atomic rearrangements even well below the macroscopic yield stress) but may not hold at stresses approaching the yield 
stress 34] and certainly does not hold if the structure is significantly altered simple structural relaxation by annealing near the glass transition temperature is sufficient to invalidate Eqn. 1 is an open question, although we note that attempts to measure volumetric thermal expansion near $T_{\mathrm{g}}$ appear to produce reasonable results [37. The basic requirements are to ensure that there is a valid, strain-free structural state as a reference and that the loading or processing history of the specimen does not alter the structure to such a degree as to invalidate the assumptions inherent in Eqn. 1.

Much of the early work focused on uniaxial tension or compression tests to establish that scattering-based strain measurements produced results consistent with other techniques. For example, in a uniaxial test the stress is known and the elastic strain can be measured according to Eqn. 1 as a function of orientation with respect to the loading direction, from which Young's modulus $E$ and Poisson's ratio $\nu$ can be determined, under the assumption that the material is elastically isotropic. The agreement between the elastic constants determined in this way and those obtained from other techniques (ultrasound or measurement of strain via extensometer or strain gage in quasi-static testing) is reasonable but there are apparently systematic differences (Fig. 4). The reasons for these discrepancies are not clear, but they are an indication that the $\mathrm{x}$-ray strain measurements are not measuring precisely the same thing as more traditional techniques.

If the strain state can be accurately measured with a scattering technique, then interesting possibilities ensue. For example, one can measure the local strain in specific regions of a sample under complex loading. Fig. 5(a) shows the von Mises effective stress around a mode I crack tip in a metallic glass. Part (b) of the figure shows that the stress falls off as the inverse square root of the distance from the crack tip, as predicted by linear elastic fracture mechanics. Near the crack tip this relationship does not hold because the x-ray strain-stress relationship becomes nonlinear, as mentioned above. 
Figure 4: Agreement of (a) Young's modulus and (b) Poisson's ratio between x-ray strain measurements and measurements from other techniques (ultrasound, extensometer, or strain gauge). Data from references [33, 34, 38, 45]. 
(a)
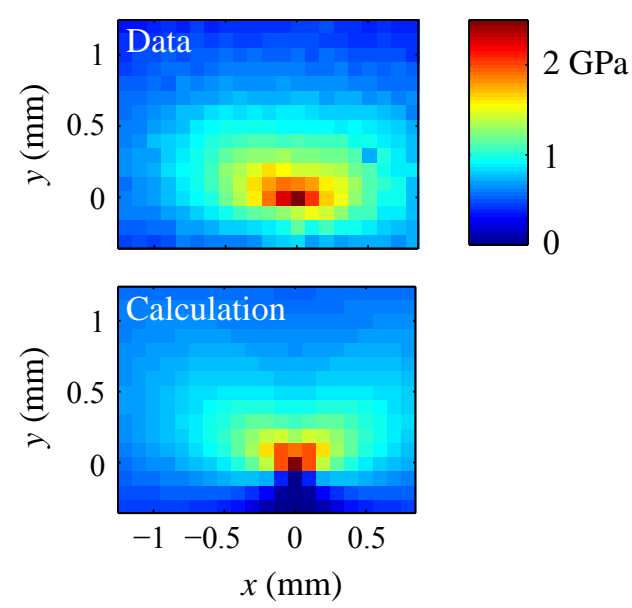

(b) Distance from crack tip, $r(\mathrm{~mm})$

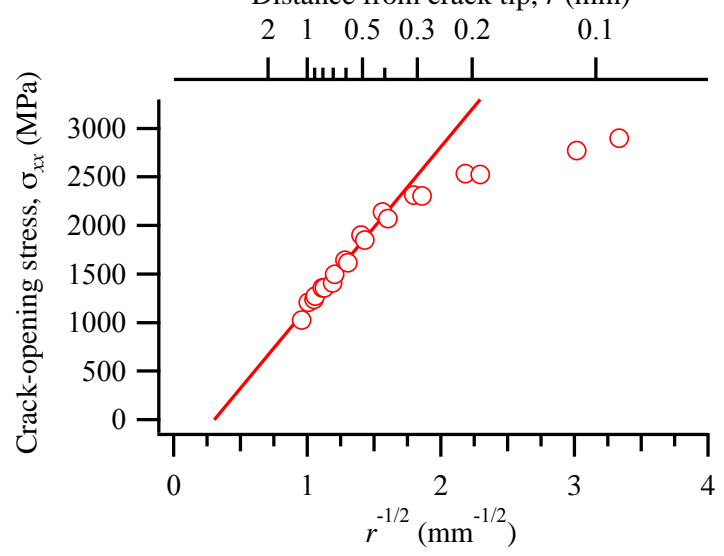

Figure 5: (a) Crack-opening normal stress $\left(\sigma_{x x}\right)$ around a mode I crack in a single-edge notch bend specimen of a Zr-based metallic glass at a stress intensity of $K_{\mathrm{I}}=47 \mathrm{MPam}^{1 / 2}$ measured with high-energy x-ray scattering, compared to a linear elastic calculation. (b) $\sigma_{x x}$ as a function of distance ahead of the crack tip $(r)$ at $K_{\mathrm{I}}=76 \mathrm{MPam}^{1 / 2}$. Far from the crack tip $\sigma_{x x}$ falls off as $r^{-1 / 2}$ as predicted by fracture mechanics. The deviation from $r^{-1 / 2}$ behavior close the the crack tip is due to plasticity and non-linearity of the x-ray strain measurement. From Ref. [32] and used under the terms of the Creative Commons Attribution License. 


\subsection{The influence of non-affine atomic displacements on elastic response} malize the scattered intensities $I(q)$ to produce the total structure factor $S(q)$, and Fourier transform $S(q)$ to get the pair distribution function, $\rho(r)$. The peaks in $\rho(r)$ correspond to the distances of atomic coordination shells around an average atom in the material, so shifts in these peak positions due to me-

These non-affine atomic displacements affect the mechanical response of metallic glasses. For example, it has long been recognized that metallic glasses are more compliant (less stiff) than crystals of similar composition (Fig. 6] [48, 
215
49]. The additional compliance is inherent to all non-crystalline solids and is a reflection of the disordered structure [50 52. In a homogeneous structure, a homogeneous strain necessarily results in a homogeneous stress and the elastic constants can be derived in terms of derivatives of the atomic potential energy with respect to strain; the result is commonly called the Born contribution to the elastic constants. (This statement is strictly true only at zero temperature because it ignores entropic contributions which may become important at finite temperatures.) In an inhomogeneous structure, on the other hand, a homogeneous strain results in an inhomogeneous stress and non-affine displacements of the atoms can reduce the internal energy of the solid. This results in a negative contribution to the elastic modulus, reducing it relative to the Born contribution alone [50]. The heterogeneity of non-affine displacements is a reflection of the fundamentally heterogeneous structure of amorphous solids and has been explored in simulations of a variety of systems, including metallic glasses [15, 53, two- and three-dimensional Lennard-Jones glasses [54, 55], silica [56, amor230 phous polymers [52, and granular materials [57]. Typically these simulations show deviations from Hooke's Law on short length scales (the particular length scale depending on the system being studied) and a strong heterogeneity of elastic response on intermediate length scales. Only at longer length scales does the response become homogeneous and linear.

235 An important direction for research is to move from these general considerations towards an improved understanding of the relationship between specific aspects of the structure and the resulting mechanical response. For example, a strong contribution of non-affine relaxation to the elastic response is apparent in three-dimensional simulations of $\mathrm{Cu}_{64} \mathrm{Zr}_{36}$, in which it has been further observed that the non-affine contribution to the elastic response varies dramatically with the preparation of the glass, particularly the rate of quenching [15]. This particular glass exhibits a high degree of icosahedral short-range order, and regions of high modulus correlate with icosahedrally coordinated $\mathrm{Cu}$-centered clusters. 

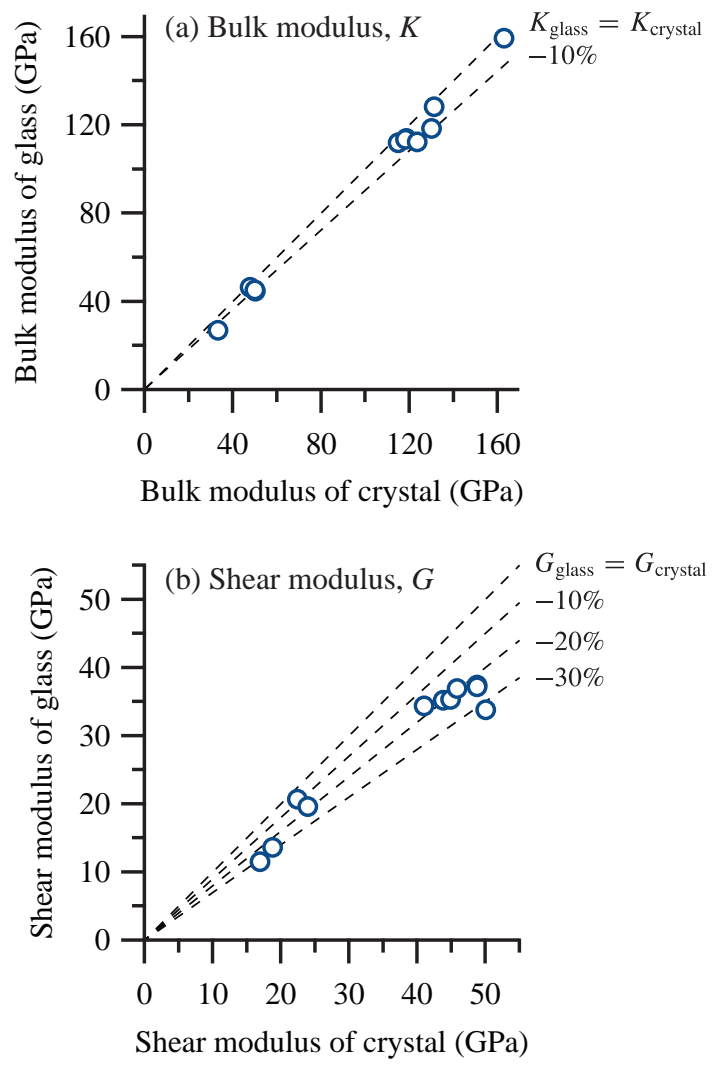

Figure 6: (a) The bulk modulus $(K)$ of a metallic glass is typically some $5-10 \%$ lower than that of a crystal of the same composition, but the shear modulus (b) is $20-30 \%$ lower. Data from Ref. 9] 


\subsection{Mechanical instabilities and the potential energy landscape}

The disordered, heterogeneous structure of metallic glasses suggests that we should expect a complex variety of atomic rearrangements in response to externally-imposed displacements. Some of the non-affine rearrangements described above, which occur at small strains, are clearly non-dissipative - they are fully reversible without the dissipation of energy as heat. In these rearrangements atoms of different types continuously adjust to their local environments as the material is subjected to remote strain. As the strain increases, however, it is clear that at some point the structure does undergo dissipative rearrangements, involving either local mechanical instabilities (particularly at low temperatures or in the ideally athermal limit) or thermal activation.

In understanding the connection between structure and deformation processes in amorphous materials, some familiarity with the concept of a potential energy landscape is helpful. The potential energy landscape is simply the many-body potential energy of a material, expressed as a function of the atomic positions [58, 59]. Figure 7(a) is a schematic one-dimensional potential energy landscape for a glass, which has many possible atomic configurations. At low temperatures we expect to find the system near one of the local minima in the potential energy landscape, which are called "inherent structures" or, more colloquially, "basins." With increasing temperature the system can explore nearby inherent structures via thermal activation over the saddle points connecting them. Transitions of this sort among closely-related configurations are associated with the fast $\beta$ relaxation (inset to Fig. 7(a)).

The potential energy landscape of a glass-forming material also has largerscale topography, described in terms of "metabasins" (or sometimes "megabasins"). 270 Upon cooling through the glass transition a glass will find itself in an inherent state $(A$ in the inset to Fig. $7(\mathrm{a}))$ in one of these metabasins. Although the glass can explore nearby configurations $(B$ and $C$ ) via the $\beta$ relaxation process, the asymmetry in barriers means that $A \rightarrow B$ transitions are more likely than $A \rightarrow C$ transitions. The system will therefore remain in the same metabasin unless a sequence of transitions to higher-energy inherent states occurs, allow- 
(a)

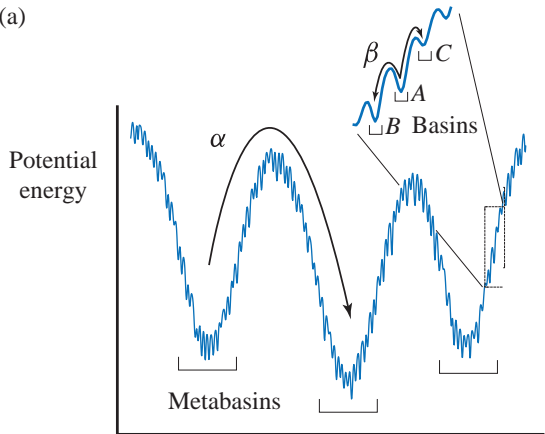

Configurational coordinate (b)

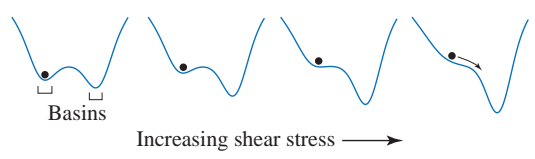

(c)

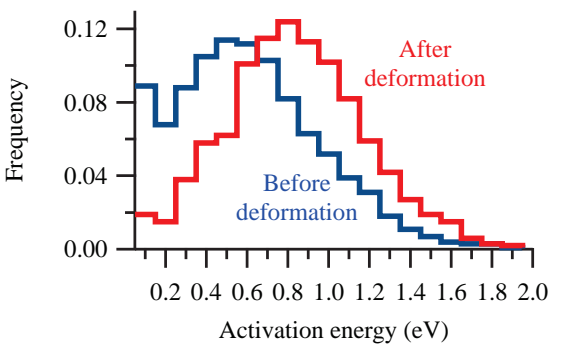

Figure 7: (a) Schematic potential energy landscape for a glass. Upon cooling from the liquid, the system will find itself in one of the basins ( $A$ in the inset.) The $\beta$ relaxation corresponds to a thermally-activated transition to a neighboring basin $(A \rightarrow B)$ but a glass does not have enough thermal energy to transition to another metabasin by a series of uphill jumps $(A \rightarrow C)$. Changing to another metabasin requires sufficient thermal energy to activate the $\alpha$ transition - that is, the glass must be heated through the glass transition to become a supercooled liquid again. (b) Applying a shear stress changes the shape of the potential energy landscape, possibly rendering some stable configurations unstable by eliminating the barrier to a neighboring configuration. (c) Distribution of activation energies in a simulated metallic glass before and after plastic deformation, from Ref. [60]. 
ing it to escape to an adjacent metabasin. This requires substantial thermal activation and is the basis of the slower $\alpha$ relaxation process.

For a given collection of atoms there is only one potential energy landscape, since the configuration parameter describes all $3^{N}$ possible locations of $N$ atoms, regardless of whether the material is a liquid or a glass. Which state a material is in depends on its temperature, because temperature determines the dynamics with which the material explores the potential energy landscape. Liquids move easily between adjacent inherent states, and even to different metabasins. Glasses are essentially frozen into an inherent state, moving to a new inherent state only infrequently via a $\beta$ relaxation process and never to another metabasin (on the time scale of the observations).

Atomic rearrangements associated with mechanical deformation correspond to traversing saddle points in the potential energy landscape and are connected to the two major types of relaxation processes in glasses. The primary (or $\alpha$ ) relaxation reflects the dynamics associated with the glass transition, when a supercooled liquid vitrifies to become an amorphous solid. The secondary (or $\beta$ ) relaxation corresponds to atomic rearrangements on a smaller scale and is the main source of dynamics below the glass transition temperature [59, 61]. At one time it was thought that the $\beta$ relaxation was only associated with intramolecular degrees of freedom (such as rotation of side groups in polymer glasses), but Johari and Goldstein showed that $\beta$ relaxation occurs even in glasses composed of rigid molecules 62. This, together with the observation of $\beta$ relaxation in metallic glasses [63] (where no intramolecular degrees of freedom exist) clearly shows that this category of relaxation must be associated with collective rearrangements of atoms or molecules.

The connection to mechanical behavior arises from the effect of deformation on the potential energy landscape [64, 65]. In particular, shear deformation can cause minima in the potential energy to disappear, rendering the system unstable and forcing it to move to an alternative nearby inherent state (Fig. 7(b)). Simulations suggest that a relatively small number of atoms (10-50) participate in the mechanical instability [64, 66]. It seems clear that since both these 
mechanical instabilities and the $\beta$ relaxation are strongly influenced by the potential energy landscape around the particular inherent state of the glass, the two phenomena must be related in a fundamental way.

The atomic rearrangements associated with the mechanical instability of the potential energy landscape are typically called "shear transformations" following the seminal bubble raft investigations of Argon and coworkers [67, 68]. Some controversy has arisen regarding whether sites of potential shear transformations are somehow encoded in the structure of the glass. Those who hold such a viewpoint typically refer to these regions as "shear transformation zones" (STZs). If this view is correct then one could, in principle, quantify the population of STZs in some way that would describe the mechanical state of the material.

Regardless of whether sites for shear transformations are intrinsic to the structure, shear transformations occur not just in the plastic regime but quite early on in the portion of deformation typically characterized as elastic. In this regard they can result in small but measurable amounts of dissipative reversible response even at quite low strains [69. This could arise either due to the imposed strain lowering a barrier so as to permit thermal activation, or by introducing an instability that sends the system into a lower energy state which may or may not be stable in the unstrained state. At finite temperatures this leads to rate-dependent elastic behavior-viscoelasticity or internal friction that can be measured experimentally [70]. At larger strains the shear transformations lead to irreversible plastic deformation events, as described in the next section.

\section{Plastic deformation}

\section{4.1. Shear transformations}

One connection between the anelastic response discussed in the preceding section and plastic deformation is a recent analysis of dynamic mechanical response, revealing evidence of distinct energies associated with STZs of discrete sizes [1] 73]. This suggests a picture in which the operative STZ sizes are determined by the loading rate or, equivalently, the applied stress. For a particular 

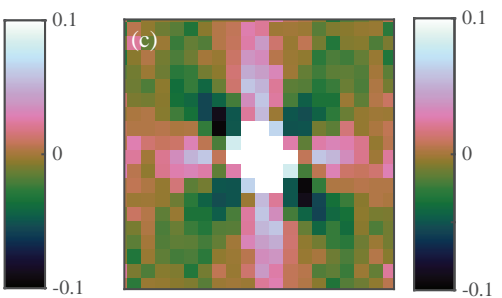

Figure 8: Quadrupolar strain fields around STZs in a colloidal glass. (a) Calculated shear strain field around an STZ modeled as an Eshelby inclusion subjected to a strain of 0.05. (b) Self-correlation function of the strain from (a), normalized to the maximum value of the self-correlation and with a color axis set to show only values of $0 \pm 10 \%$ of the maximum. (c) Experimentally measured self-correlation of the strains in a colloidal glass at a strain level similar to that in (a) and (b). (Calculations and data from Ref. 85].)

loading rate there will be a particular STZ size below which the STZs can relax quickly compared to the loading rate and thus contribute to the anelastic response, while larger STZs are essentially frozen in their initial configuration until the stress becomes large enough to trigger the rearrangement. Thus, while it appears that there is a broad distribution of STZ sizes, the plastic response appears to be dominated by the portion of the distribution just beyond the reach of thermal activation on the experimental time scale.

Quite a bit of work has been done to characterize the sizes and energies of shear transformation zones by atomistic simulations [74 [78, experiments on metallic glasses 79 83, and experiments on model systems, especially colloids [84, 85]. For example, Fig. 8 illustrates the shear strain field around an STZ modeled as an Eshelby-type inclusion, compared to measurements on a sheared colloidal glass. The quadrupolar nature of the strain field around the inclusion is clearly reflected in the local displacements of the colloid particles.

Several controversies about shear transformations remain, however. One issue regards the nature of the structural features that play roles analogous to dislocations in crystals. Both Argon's shear transformation [86] and Spaepen's flow defect 87] were conceptualized as localized plastic rearrangements. Atom- 
istic simulations, particularly those performed in the flowing steady state at 5 correlations in the deformation events $[76,88,90$. These ramified atomic rearrangements are prone to misidentification as shear bands, but they are different from experimentally-observed shear bands in metallic glasses in that they are transient and typically do not persist long enough to localize a significant strain. Interestingly, they have quite profound effects on shear induced diffusion in the glass that can be quantified to extract a dimensionality for these collective events 91]. The main controversy around these observations is whether such ramified correlated events are more characteristic of the fundamental process by which glasses deform than the localized regions of deformation envisioned by 365 Argon [86], Spaepen [87] and others.

More recent investigations have revealed that even these larger events can be thought of as localized rearrangements that exhibit the typical quadrupolar stress signature of an Eshelby inclusion [76. It has been hypothesized that the long-range elastic interactions by which such objects interact may induce these localized STZs to correlate in space and time 92. This is likely to be more pronounced in steady-state shear where mechanical deformation has strongly disordered the structure of the material and the STZ density (or susceptibility to shear transformations) is consequently high. In addition this trend may be strongly influenced by inertial effects 93 .

Another intriguing question links the elastic and plastic response of metallic glasses: Does the linear elastic response of a metallic glass, and in particular the phonon spectrum at low frequencies, provide any information about the sites of plastic deformation? Schober and coworkers associated low-frequency vibrational modes with $\beta$ relaxation and demonstrated that these modes are a combination of plane waves and localized vibrations [94 97. More recent work suggests a correlation between atoms that participate strongly in low-frequency phonon modes and deformation activity, with such atoms being several times more likely than a random atom to participate in deformation. Regions containing such atoms have been denoted "soft spots" and they appear to persist on 

415 tions in their vicinity [98, 99. This is surprising because the vibrational modes being probed are essentially aspects of the elastic response of the glass that say nothing directly about the saddle points connecting the inherent states prior to and after the activation of an STZ. It is an open question as to whether these soft spots are STZs or whether it is better to think of them simply as "fertile regions" that can give rise to shear transformations. An important recent result is the observation that such regions appear to be susceptible to deformation only in certain orientations [100, indicating that STZs really are encoded in the structure of the glass and that the "fertile region" interpretation may be insufficient. The reason for this close linkage between linear response and the available structural transitions remains unclear, however.

There is now abundant evidence that a variety of properties of metallic glasses are heterogeneous on the nanometer scale; this include stiffness [101, 102, yield load in nanoindentation [103, and energy dissipation [104]. The structural heterogeneity also means that the mechanical properties can be anisotropic on the nanoscale 105. Recently, Ketov and coworkers showed that thermal cycling to low temperatures induced structural rejuvenation of a metallic glass [106]. They attributed this to the thermal expansion coefficient being heterogeneous on the nanoscale, and it may be that the thermal expansion is anisotropic as well. The variation in thermal expansion causes local stress concentrations upon heating or cooling and thus irreversible non-affine atomic rearrangements, which in turn affect the subsequent deformation behavior of the glass. It has also been asserted that the structure of metallic glasses may have a significant volume fraction (perhaps a quarter of the total) for which the structure resembles that of the liquid even more closely than does the structure of the remainder of the glass [107]. Presumably the length scale of the heterogeneity is small, although this was not directly accessible in that study.

These observations point out a fundamental distinction between glasses and crystals: Crystals are, for the most part, uniform in their structure but glasses 
crystals is of course essential for understanding mechanical behavior, but these defects can conveniently be viewed either as perturbations on the underlying uniform structure or as independent entities in their own right. An important question is whether distinct structural defects are important for understanding the behavior of glasses, where the structure is heterogeneous by its very nature and there is a distribution of structural states.

\subsection{Structural evolution under load: Constitutive equations}

Another area of active research, at the intersection of materials science and continuum mechanics, is the development of broadly applicable constitutive equations suitable for modeling the evolution of metallic glass under various mechanical loads and thermal histories. Early work by Argon [86 and Spaepen 87 . proposed generalized constitutive forms for the activation of shear transformations or flow defects respectively, which were subsequently applied in an attempt to understand the instability that leads to strain localization (and particularly whether the instability was due to structural softening or heating) [108 110.

Any attempt to develop a generalized constitutive model faces the problem of how to describe the evolution of the structure of the glass with time. Much early work was based on the free volume model of Cohen and Turnbull [111, 112, implicitly assuming that free volume is a natural description of metallic glass structure. The viscoplastic methodology of Anand and $\mathrm{Su}$, for example, relies on free volume and another empirical parameter, cohesion, which evolve monotonically towards steady-state values [113. Shear softening and localization are observed in this model (Fig. 9) but the scale of the shear bands is limited by the finite-element mesh because there is no internal length scale. Qualitative agreement with experiments and atomistic simulations is observed [114, but like other free-volume-based methods no clear means exists to validate the internal parameters against experimentally measurable or atomistically simulated details. The model has also been used to model experimental behavior at high homologous temperatures [115] but runs into issues at low temperatures because 

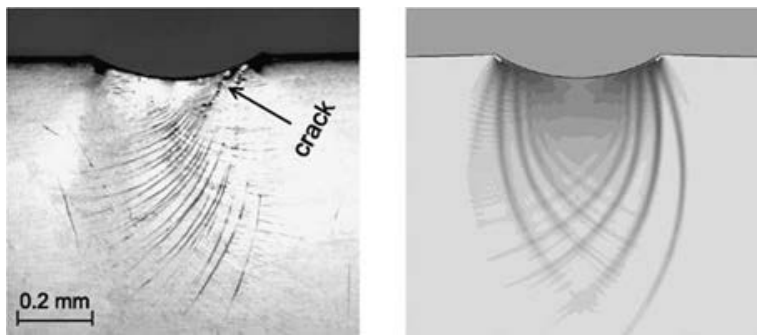

Figure 9: Comparison between experimentally-observed shear band patterns resulting from plane-strain indentation of a metallic glass (top) with a contour plot the plastic strain (bottom) calculated using the constitutive model of Su and Anand [114. (Figure from Ref. [114] and used by permission.)

ficulty of dealing with rate sensitivity it also removes any explicit connection between flow and structure. Attempts have been made to address this problem by instead incorporating a variation on the Peierls concept [116] but it is not clear this is appropriate, given that coarse-graining of STZ structure is an assumption of all such constitutive modeling.

Johnson, Lu and Demetriou [117] have proposed a free-volume-based theory for deformation and flow at high homologous temperatures. This theory relies on a self-consistent methodology and assumes disordering at a rate proportional to the plastic strain rate in competition with relaxation on time scales described by the Vogel-Fulcher-Tammann (VFT) equation. Although semi-empirical, the VFT relation gives a good fit to viscosity measurements over a wide range of temperatures in equilibrium and supercooled liquids. The resulting theory provides excellent fits to the emergence of non-Newtonian behavior in the liquid at low temperatures and high strain rates. It can also provide a means of calculating the strain rate sensitivity, which is important to evaluating the stability of flows against localization. However, the divergence of the relaxation time as one approaches the VFT temperature (which is typically significantly above room temperature) clearly limits this theory to the liquid and supercooled liquid regimes.

A different methodology which also builds upon the prior work of Argon and 
Spaepen is the effective temperature STZ theory [118]. Originally developed in the context of direct comparison with low-temperature molecular dynamics simulations of glasses subjected to different stress and strain controlled mechanical loads [119, this methodology attempts to build a mean-field theory around the STZ concept of defects. The main deviation from the Argon theory is the proposition that the STZ population can be used to describe the structural state of the system. The main deviation from Spaepen's flow defect picture is that the orientations of the defects as well as their number density are taken into account in a way that allows for the existence of a clear transition from bounded to unbounded flow at a well-defined flow stress.

Recent developments embedding the STZ theory within an effective temperature formulation have placed the theory on a thermodynamic foundation distinct from the free volume hypotheses of Turnbull and Cohen [120, 121]. In this formulation the slow structural degrees of freedom of the glass are described as a distinct sub-system separable from, and only weakly coupled to, the fast, primarily vibrational degrees of freedom that come into equilibrium with the environment on short time scales. The disorder in the structural sub-system is described in terms of its configurational entropy and energy. Since the derivative of an energy with respect to an entropy is a temperature, albeit one that may be far out of equilibrium, this permits the formal definition of an effective temperature of the structural degrees of freedom. The evolution of this effective temperature must be explicitly modeled. This equation of motion is far more constrained than the evolution law for free volume because it must represent how the mechanical work on the structural sub-system raises the energy - that is to say, it is an expression of the first law of thermodynamics. Furthermore, the second law of thermodynamics requires that the rate of dissipated plastic work is non-negative after one takes into account the fraction of potentially recoverable work, such as the work stored in anelastic deformation and defect generation. This provides guidance on how the evolution of the structural state couples to the rate equations proposed to describe the kinetics.

The assumptions of the effective temperature theory have been validated by 
comparing aspects of the constitutive equation to atomistic simulations. In particular, molecular dynamics simulations of shear bands in simple 2D LennardJones systems have permitted a test of the assumption that (in at least some cases) one can separate the effect of the effective temperature on flow from the effect of stress on the STZ transition rates [122]. Furthermore, numerical implementations of the effective temperature STZ theory facilitate understanding of the development of shear bands near stress inhomogeneities [123, 124] and cavitation near crack tips (Fig. 10] [125]. The theory has also been successfully applied to explain experimental investigations of materials annealed for varying times at stresses close to yield [126, 127, rheology at various temperatures near the glass transition [128, experiments regarding variable amplitude cyclic loading [129], and stress overshoots during serrated flow [130].

\section{Localized plastic deformation: Shear bands}

The tendency for metallic glasses to experience shear localization at temperatures well below the glass transition due to a lack of strain- and strain-rate hardening has long been recognized [5, 131]. Two excellent review articles on shear bands in metallic glasses have recently appeared [10, 132] so in this section we highlight some recent results of particular importance and seek to place them in context. Because terminology regarding shear bands is often imprecise, we adhere to the language proposed in Ref. [5]:

- shear front refers to a propagating front of shear displacement;

- shear banding event refers to the initiation and propagation of localized shear displacement, whether progressively as a shear front or by uniform deformation on a shear band;

- shear band refers to the approximately planar volume of material that is sheared during a shear banding event.

\subsection{Nature of shear banding events}

Greer, Cheng, and Ma [10] helpfully outlined three scenarios for shear band525 ing events. The first is homogeneous nucleation, in which STZs activated 
Figure 10: Numerical solution of STZ equations reveals differences in stress state (shown here as the hydrostatic stress $p$ normalized to the yield stress in shear, $s_{\mathrm{y}}$ ) ahead of a crack tip, depending on the structural relaxation of the glass as quantified by the effective temperature $\chi_{0}$. At the same overall stress intensity the stress concentration is more pronounced in a morerelaxed glass (a) than in a less-relaxed glass (b), and the crack tip morphology is different as well. These differences lead to cavitation in the more-relaxed glass (c) and ultimately to catastrophic failure (d). The crack tip morphology in (d) is similar to that observed experimentally (e). (Data for parts (a)-(d) from Ref. [125]. Part (e) is from Ref. [32] and used under the terms of the Creative Commons Attribution License.) 
throughout the material by an imposed stress link up when the percolation threshold is reached to form a shear band. Disruption of the atomic- or mediumrange order on this band can lower the flow stress and allow continued uniform sliding on the band. As one might expect, the stress required for homogeneous nucleation is large [133] and so although it is commonly observed in atomistic simulations [134, in real materials it is likely that shear banding events initiate heterogeneously at stress concentrations, as seen in simulations with inhomogeneous loading geometries [135]137.

A second scenario involves initiation at a stress concentration followed by propagation across the specimen of a mature shear front that carries the entire shear offset associated with the shear banding event (akin to a mode II shear crack). One model of this behavior, by Shimizu and coworkers [138, made several specific predictions, including that the mature shear front would propagate at approximately the shear wave speed of the glass and that it would be accompanied by significant temperature increases, to at least the glass transition. Although this model may be appropriate for high-rate loading, it is now known that shear banding events under quasi-static loading occur over time scales much longer than those predicted by this model [139 142] and that, at least in some situations, shear banding events occur without large temperature increases [143, 144]. Furthermore, it is not clear how this model can accommodate situations in which there is a variation in shear strain along a shear band, for example in bending [145] or near a mode I crack tip [146].

It appears likely that under quasi-static loading shear bands form by initiation of a shear front at a stress concentration. The shear front propagates across the specimen, disordering the atomic-scale structure along its path, lowering the local flow stress, and allowing continued deformation on the resulting shear band. Under a macroscopically homogeneous strain state (such as uniaxial compression or tension) continued deformation on the shear band can occur by sliding along the entire length of the shear band simultaneously, as has been observed with high-speed cinematography [142, 147]. This picture is consistent with scanning-electron microscopy (SEM) observations of fracture surfaces 
that show no difference between the two ends of the shear band [10, 148]. Under other loading conditions such as bending there can be a gradient in shear offset along the length of the shear band. For example, $\mathrm{Qu}$ and coworkers recently showed that shear bands that form at the macroscopic yield stress and span the specimen have shear offsets that are independent of position along the band [149], presumably because the loading conditions permit plastic deformation everywhere along the band simultaneously as just described. On the other hand, they also observed that shear bands that form below the macroscopic yield stress and do not span the specimen have shear offsets that vary with position along the band. The latter case presumably arises when a shear front initiates at a stress concentration and propagates some distance away, but away from the stress concentrator there is insufficient driving force to allow continued propagation.

It has been proposed that the reduction in viscosity or flow stress associated with shear banding events is due to a stress-induced glass transition, in which the shear stress disrupts the atomic-scale structure of the glass so as to become more like the equilibrium structure in the liquid [150. Indeed, it has been recently observed that there is a brief $(\sim 10 \mu \mathrm{s})$ burst of acoustic emission around the onset of a load drop during serrated flow, quantitative analysis of which suggests that it is associated with a dilatation of about $2 \%$, comparable to that which would be observed on heating to the glass transition [158, 159. Heating due to plastic work in the shear band can reduce the flow stress or viscosity, but it is now widely accepted that this is typically a consequence rather than 580 a cause of plastic deformation [5, 10, 137, 151]. It is important to bear in mind that heating is not an intrinsic property of shear bands, but is sensitive to extrinsic conditions including the imposed strain rate, the size of the sample, and the stiffness of the testing machine [152]. Nevertheless, in at least some cases there is little heating associated with quasi-static deformation because the time scale over which heating occurs (a few milliseconds) is comparable to the characteristic time scale for thermal diffusion $\left(\tau=l^{2} / \alpha \simeq 3 \mathrm{~ms}\right.$ for a diffusion distance $l=100 \mu \mathrm{m}$ and thermal diffusivity $\alpha=3 \times 10^{-6} \mathrm{~m}^{2} \mathrm{~s}^{-1}$ 
for Vitreloy 1, 153]) 143, 144. There is no contradiction here with the longstanding observation of significant melting on fracture surfaces because fracture occurs on much shorter timescales (in a matter of microseconds) that allow significant heating [144] (Fig. 11). The difference in time scales implies that unstable fracture of metallic glasses is more than a simple runaway shear banding event on a single shear band. One clear difference is that fracture involves cavitation [154, 155], a subject that has recently been the focus of several computational studies [125, 156, 157].

The inhomogeneous mode of deformation by shear banding events gives way to homogeneous deformation as the testing temperature approaches the glass transition (see Fig. 19 in Ref. [5]). One controversy in the literature has been whether a transition from shear banding to homogeneous deformation also occurs as a function of sample size. Around the time of Ref. [5] several groups reported that sub-micron specimens deform homogeneously [160-162] while other groups reported shear banding even for samples as small as $150 \mathrm{~nm}$ [163, 164]. The literature on this subject and possible explanations for the apparent transition and the disparate results have been extensively reviewed [10, 165]. Here we draw attention to the recent work of Tönnies and coworkers, who reported that the spacing of shear bands was proportional to the diameter of their specimens, and suggested that the transition to homogeneous deformation occurs when the spacing of the shear bands becomes comparable to their thickness [166]. This explanation avoids the necessity of invoking a length scale for the transition based on a presumed nucleation size for shear bands.

From a theoretical perspective, another means of analyzing shear bands and inhomogeneous deformation has been to implement constitutive equations such as the effective-temperature STZ equations described in Section 4.2 above. Simulations of a stretched bar with a notch utilizing such a methodology have shown that in such models shear bands can grow quasi-statically from stress concentrations due to the feedback between plastic flow and disordering that arise in the material [123] and produce results similar to molecular dynamics simulations [135] and experiments [139, 142. These methods have also provided 

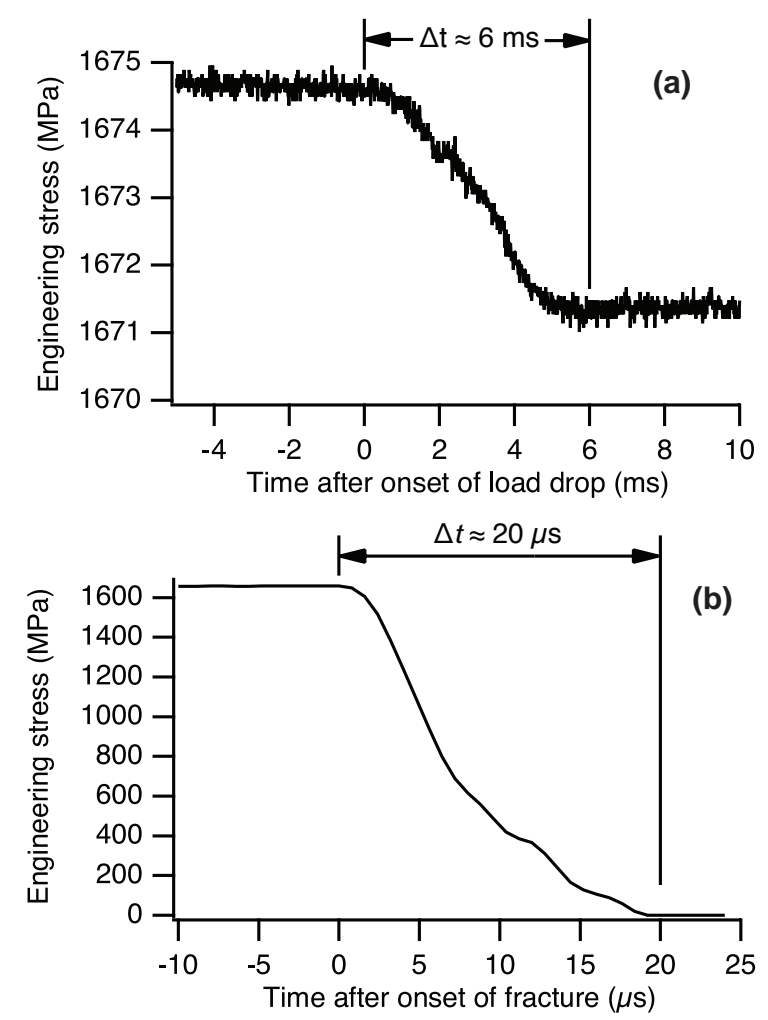

Figure 11: Fracture of metallic glasses in uniaxial compression occurs on much shorter time scales than shear band operation. The load drop due to a shear banding event evolves over several milliseconds (a), while fracture is over in a matter of microseconds (b). Figure reproduced with minor formatting changes from Ref. 144] under the CC BY 3.0 license. 
quantitative means for analyzing the waiting time, strain rate, and temperature dependence of serrated flow in shear bands in metallic glasses [130].

\subsection{Structure and properties of shear bands}

The large shear strains in shear bands suggest that their structure should be different from the surrounding material, which is either undeformed or at least not as extensively deformed. Transmission electron microscopy (TEM) would appear to be an ideal choice for investigating the structure of shear bands. Most TEM studies agree that the thickness of shear bands in metallic glasses is around 5-20 nm [10, 167]. In many such studies the shear band region was thinner than the surrounding material making interpretation problematic [168, 169, but a recent study using a focused ion beam (FIB) milling lift-out technique to produce TEM specimens of uniform thickness yielded results similar to the earlier work [170].

A more fundamental problem is that the thickness of a shear band, and the structural evolution of the material within it, reflect the conditions under which it was created. An early cautionary note was provided by the observation that both the dilatation and stored energy of extensively deformed metallic glass ribbons is too large to be attributed to shear bands of this thickness, if it is assumed that plastic deformation occurs only in shear bands that leave slip steps on the surface of the specimen [171, 172]. This suggests either that shear bands are thicker than the TEM observations cited above indicate, or that plastic deformation occurs outside the shear bands.

Fundamentally, plastic deformation of any kind is a response of the sample to the displacements imposed on it. For metallic glasses the imposed displacements during plastic deformation well below the glass transition can be accommodated by shear bands ranging from single bands with extensive shear [173, 174] to a 645 profusion of bands with minimal shear [175] or even homogeneous deformation [126, 127, 176, depending on the sample geometry and loading conditions. The most common approach to TEM studies is to form shear bands by bending thin specimens. Conner and co-workers showed that the displacement on shear 
bands in a plate loaded in simple bending increases as the square of the thickness of the plate 177,178 . Although the shear strain is probably not uniform across a shear band, it is reasonable to expect that the shear band thickness $t$ may be proportional to the displacement $u$ through $t=u / \tan \bar{\gamma}$ where $\bar{\gamma}$ is the average shear strain in the band. If this is true then the small displacements associated with bending a thin TEM specimen would result in thin shear bands.

There are, in fact, indications that shear bands produced in bulk specimens are thicker than those seen in TEM. For example, experiments in which a series of nanoindentations are made across the thickness of a shear band do reveal reductions in hardness 173, 174 and modulus [174, over comparatively large distances (Fig. 12). On the other hand, surface traces reveal no evidence of significant plastic flow so far from the center of the shear band (see for example the images in Ref. [144]), and a stability analysis of the differential equations governing coupled thermal and dilatational softening suggests that the characteristic thickness of shear bands should be on the nanometer scale if the softening is dominated by free volume effects [179. It is therefore unlikely that changes in hardness and modulus over distances of a micrometer or more are a direct manifestation of structural changes due to plastic flow within a thick shear band. Far from the center of the band the plastic flow is likely quite low and would tend to appear more like a homogeneous background flow. It would not require especially large strains to affect modulus or hardness if the atomic environments were significantly disturbed, tilting the balance of the different types of clusters towards those that are GUM-like (Sec. 2). Alternatively, changes in hardness and modulus away from the center of the band may be due to residual stresses developed around a narrow shear band during deformation, or to diffusive structural relaxation after the deformation ceases.

Although atomistic simulations indicate that the extensive shearing inside a shear band disrupts the atomic short-range order of the glass [122, 134, 137, 180, detailed experimental evidence of structural evolution in shear bands is surprisingly scarce. The older literature is reviewed in Ref. [5]. More recently, it was found that shear bands formed by compression of a bulk specimen, and 


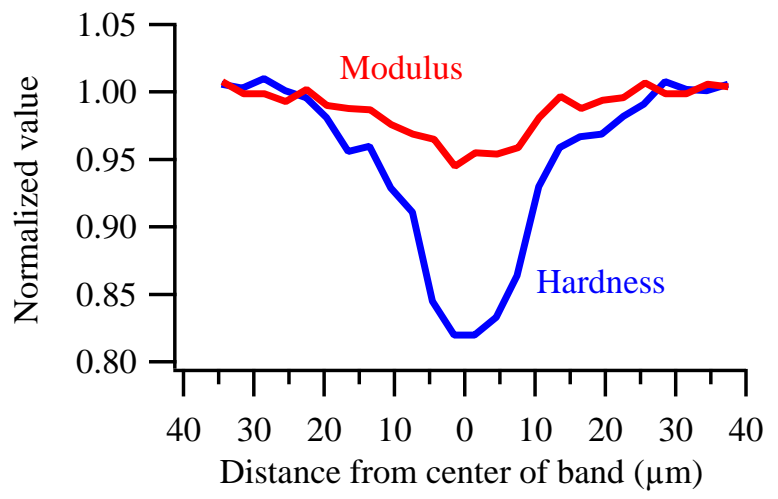

Figure 12: Hardness and modulus across a single shear band measured by nanoindentation, normalized to the values well away from the shear band. Significant effects can be observed as far as $20 \mu \mathrm{m}$ from the center of the band. (Data from Ref. 174].)

TEM images, although small changes in electron scattering patterns were observed [168. However, a lift-out technique applied to thin ribbon specimens did yield clear contrast in high-angular dark field scanning-transmission electron microscopy images as well as electron energy-loss spectroscopy images, which were attributed to dilatation in the shear bands [170. Similar techniques on thin-film samples prepared by electropolishing have been used to infer density variations along the length of shear bands 181. Other recent evidence for dilatation in shear bands include density changes in cold-rolled glasses [182, enthalpy recovery from rolled specimens [182, 183, and relaxation of dilatation in a single shear band via thermomechanical analysis [173]. The observation of dramatically enhanced atomic diffusivity [184] and a preference for crystallization in shear bands [185] are also suggestive of structural disorder. Finally, we note that there is also evidence for more complex shear band structures, such as distinct inner and outer zones [186].

Broadly speaking, the experimental observations seem to be consistent with the simulation results but more work is needed to clarify the details of structural differences between shear bands and the surrounding material. It should 
be noted that a highly disordered structure during a shear banding event implies rapid structural relaxation once deformation ceases, so the static structure

measured after the fact in experiments provides only indirect information about the flowing state. True in situ studies of structural evolution inside shear bands would be desirable. These present a formidable challenge at room temperature due to the short time scales involved, but they may be possible at cryogenic temperatures where single shear bands that evolve over many seconds can be produced [187.

\subsection{Dynamics of shear banding events}

From a practical point of view, the dynamics of shear banding events determine whether shear banding can be a dominant mode of plastic deformation, and probably also have a strong influence on the rate dependence of fracture toughness. More fundamentally, although the fundamental mechanism of deformation (shear transformations) is believed to be the same for both homogeneous and heterogeneous deformation, the time and length scales associated with shear banding events at room temperature make experimental studies on the atomic scale difficult. But because these mechanisms (along with the loading conditions) determine the shear band dynamics, careful measurement and understanding of the dynamics can provide insights into the mechanisms of deformation.

Perhaps the most familiar manifestation of shear band dynamics is the appearance of abrupt load drops during either confined or displacement-controlled uniaxial compression, commonly referred to as serrated flow [188, 189]. In general, serrated flow can occur when the strain rate sensitivity of a material is negative because localized flow can cause the instantaneous strain rate of the material to exceed the imposed strain rate from the machine. This is symptomatic of dynamic strain aging in which the rate of plastic deformation is comparable to the rate of a thermally-activated relaxation mechanism over a limited range of strain rates and temperatures [190]. A prominent example is the Portevin-Le Chatelier effect in crystalline alloys in which mobile dislocations 
Figure 13: Serrated flow (filled symbols) occurs so long as the shear displacement rate $\left(v_{\mathrm{SB}}\right)$ exceeds the displacement rate imposed by the crosshead velocity $\left(\sqrt{2} v_{\mathrm{XH}}\right.$, because the shear occurs at approximately $45^{\circ}$ to the loading direction). But $v_{\mathrm{SB}}$ decreases with decreasing temperature so there is a critical temperature $T_{\text {crit }}$ below which the shear displacement rate is determined by the crosshead velocity. In this case flow becomes non-serrated (open symbols) and the shear displacement rate is independent of temperature. The cross-over temperature $\left(T_{\text {crit }}\right)$ decreases with decreasing crosshead velocity. (Figure adapted from Ref. [132] using data for $\mathrm{Zr}_{52.5} \mathrm{Cu}_{17.9} \mathrm{Ni}_{14.6} \mathrm{Al}_{10} \mathrm{Ti}_{5}$ loaded in uniaxial compression from Ref. [192].)

repeatedly break free from and are re-trapped by diffusing solute atoms [191]. Phenomenologically, one would expect that serrated flow would disappear at low temperatures and at high strain rates if the thermally-activated relaxation mechanism cannot keep up with the imposed strain rate. This is indeed observed in the Portevin-Le Chatelier effect, has long been known for serrated flow in metallic glasses as well (Fig. 13) [188, and has been included on deformation maps for metallic glasses [5].

Shear band dynamics have been examined in an elegant series of experiments by Löffler and coworkers [132, who interpreted them in terms of the effective temperature formulation of STZ theory described above [130]. By adding a thermally-activated relaxation term to the rate equation for evolution of the effective temperature at thermodynamic temperatures well below the glass transition, they were able to use the STZ theory to reproduce several significant experimental results, including the transition from serrated to 
non-serrated flow at low temperatures or high strain rates.

Serrated flow in metallic glasses is one example of a much broader class of avalanche phenomena that includes Barkhausen noise, shearing of granular materials, crumpling of paper, and dislocation-mediated plastic deformation in crystals [193]. A simple mean-field model of plastic deformation views it as the result of individual slip events (shear transformations in the case of metallic glasses) that can interact elastically to trigger a slip avalanche [194, 195]. When used to analyze the statistics of serrated flow in metallic glasses the model gives good agreement with the load drop magnitudes [189, 196] and several other statistical measures of the load drops, including duration, maximum load drop rate, and the power spectrum of the load drop rates [189]. A more stringent test is that the observed dynamics of the load drops are also in good agreement with the predictions of the model [189].

\section{Strain hardening of metallic glasses}

As discussed in the preceding section, shear localization in metallic glasses demonstrates an important aspect of their deformation physics: they strainsoften. This is perhaps the most undesirable intrinsic property of metallic glasses, as it limits their malleability and ductility, contributes to unstable fracture and, at least for some glasses, results in low toughness. If it were possible to evoke strain hardening, on the other hand, remarkable improvements in properties and behavior could result.

Since Ref. [5] was published there has been considerable progress in exploring the conditions under which strain hardening may be possible in metallic glasses. Broadly speaking, the progress can be categorized into two main classes of strain hardening: (i) exhaustion hardening, which is an apparent strain hardening that spans many shear localization events, and (ii) structural strain hardening, which occurs homogeneously by local stress-induced reduction of disorder in the atomic glass structure. 


\subsection{Exhaustion hardening: Apparent strain hardening from shear band con-} straint

One kind of strain hardening phenomenon in metallic glasses is an "exhaustion hardening" that develops as strain is accumulated over a sequence of many shear band activations. The material inside a shear band strain softens dur-

775 ing a shear banding event (indeed, this is the cause of shear localization) so, if unconstrained, significant plastic strain can accumulate on a single band. On the other hand, if deformation on a shear band can be arrested then the next shear banding event may occur elsewhere upon further loading. What results is an exhaustion hardening where the most favorable (i.e. the weakest) initiation sites are the first on which shear bands form, then the next-weakest, and so on [5, 197. Even though there is no true strain hardening the apparent strength of the entire specimen appears to rise because the stress required to trigger each shear banding event is higher than the one before. In the limit where all the "weaker" sites for shear localization are used up the glass may attain the true elastic limit, which has been approached in very small samples after exhaustion of the weakest sites [198. We call this an "apparent" strain hardening because it is unlike strain hardening in crystalline metals, where the structure becomes inherently more resistant to plastic flow, opposing strain localization and therefore developing essentially homogeneously over the strained volume.

The key to exhaustion hardening lies in preventing the overall plastic strain of the specimen from being accommodated on a single shear band (or a small number of shear bands). To do so, plastic flow on the shear band must be must be arrested despite having already been triggered and being in the middle of a self-perpetuating softening cycle.

The simplest and most common way of arresting a shear band is by unloading it; removal of the shear stress driving flow will arrest shear localization. In many testing geometries, especially those that are strain controlled, shear bands naturally become unloaded when their shear offset accommodates the imposed strain on the specimen. An intuitive example is rolling: once the thickness of a 
change is mandated and the shear bands that were triggered during deformation can be arrested. Similar statements can be made about other multiaxial loading conditions such as indentation and extrusion. In fact, the earliest references to strain hardening in metallic glasses pertain to drawn wires [199, 200] where many shear bands form and are arrested geometrically. More recent work has observed the same effect in constrained compression samples, where the aspect ratio is below $\approx 1$ and thus shear bands geometrically are forced to intersect the platens [201, 202, or in severe torsion where failure is proscribed 203. As discussed above (Sec. 5.3), even in nominally load-controlled testing of specimens with large aspect ratios, dynamic effects in the loading apparatus can lead to intermittent unloading during a shear localization event.

There is also improved understanding of what constitutes an effective geometrical constraint on an active shear band. Beyond the examples of clear mechanical constraint noted above, even small geometrical imperfections such as minor misalignments or miscuts [204] or even a single void [189] in samples under nominally unconstrained loading (such as uniaxial compression) can impose a remarkable degree of constraint. Such effects can suppress failure, permit many shear bands to form, and generally lead to apparent strain hardening and extensive malleability. Taking advantage of these effects, there have been many 820 efforts to make composite materials based on metallic glasses, where the secondphase particles provide the constraint on shear band operation 205 208. Such composites can be tough, malleable, and ductile, but further discussion of them is beyond the scope of the present review.

While geometrical constraints are an important approach to the arrest of shear banding events, in other cases arrest can be accomplished under nominally unconstrained loading, apparently due to structural changes that emerge at large shear strains within the shear band. The shear-driven nucleation of crystallites in shear bands has now been widely reported and several groups have shown that this could be associated with apparent strain hardening in samples where many shear bands formed [197, 209 211. In some glasses that already contain nanocrystals the extensive plastic flow on a shear band causes the particles to 
coalesce and align, resulting in strain hardening [212]. Both the nucleation of crystalline regions and their alignment and coalescence in shear were suggested to be important in locally hardening the shear bands at such large strains; this paves the way to their arrest and triggering of secondary shear bands, such as is required for the exhaustion hardening mechanism. Additional means of energy dissipation are also available to the crystalline phases once they do appear, such as twinning or martensitic transformation [213, which can further help to slow, bifurcate, or arrest shear banding events.

If shear banding events can be arrested after finite strain, continued plastic deformation will require triggering of new events, which may occur either on existing shear bands or on newly-formed bands. In the latter case it becomes possible to produce material with a high volume density of shear bands. Examples of this include the early work on wire drawing mentioned above [199, 200] along with constrained compression, rolling, and severe plastic deformation processing [203, 214, 215]. The influence of extensive prior plastic deformation on the response of the material to subsequent testing can be complex. On the one hand, plastic deformation within a shear band disrupts the structure of the glass. This renders the material both softer and more compliant around individual shear bands [173, 174 and tests which average over both shear bands and undeformed material also show a reduction in hardness due to prior plastic deformation [201].

An alternative behavior results from exhaustion hardening and points out the importance of loading geometry. Extensive inhomogeneous plastic deformation of a metallic glass produces a material with a wide distribution of structural states. Some material likely remains virgin or nearly so; some has been transected by a single shear band; and some, lying at shear band intersections, has been multiply shorn. The wide spectrum of local strengths in the material offers ample opportunities for triggering shear banding events. If the loading geometry favors initiation on previously-existing shear bands then the strength of the material will be reduced due to work-softening in the bands, as mentioned above. But a mismatch between the loading geometry and that of the prior 
deformation can require a new set of shear bands to form. For example, although a rolled glass sustains many shear bands that are oriented to permit the shape change imposed by rolling, these bands are not necessarily oriented to facilitate deformation in other geometries. The sample might need to form new shear bands which further transect the material and its many local structural states, opening the door to exhaustion hardening. In an elegant series of experiments illustrating this principle, Takayama measured the tensile fracture stress of metallic glass ribbons heavily deformed by rolling, as a function of the angle between the tensile axis and the prior rolling direction [216]. If the loading geometry was such that plastic deformation occurred on previously-existing shear bands then the fracture stress was reduced relative to undeformed material, in accordance with expectations based on work softening in the shear bands. However, samples tested in tension at large angles $\left(>40^{\circ}\right)$ with respect to the rolling direction - such that shear banding events did not occur on the shear bands produced by rolling - showed an increase in fracture stress, even above that of the undeformed material. Takayama speculated that shear band intersections cause hardening in a manner similar to dislocation intersections in crystals, but since shear bands are not physical defects with restoring line tension it is not clear that this analogy holds. Instead, the pre-existing shear bands may tend to favor shear in directions unfavorable for the new loading geometry, potentially deflecting the new shear bands and thus geometrically hardening the glass.

More broadly speaking, the fact that glasses have a heterogeneous local structure with a complex universe of possible arrangements provides interesting opportunities for the control of shear bands. Many authors have noted that a distribution of local strengths arising from a heterogeneous structure is desirable because it promotes a distribution of shear bands in metallic glasses [209, 210, $890 \quad 217$ 219. This is an area needing additional research if we aspire to intentionally evoke exhaustion hardening by control of the local states. 


\subsection{Strain hardening of the glass atomic structure}

Whereas exhaustion hardening as described above is about the progressive shearing of different spatial regions of a bulk glass specimen, there is a second potential mechanism for strain hardening in metallic glasses that is more like conventional strain hardening as it is known in crystalline metals and alloys. The mechanism is based on mechanically-driven relaxation of the glass structure, and is a genuine strain hardening, as it opposes strain localization and therefore can occur homogeneously. For simplicity we term it "structural strain hardening" to differentiate it from exhaustion hardening.

In some sense, structural strain hardening of metallic glasses has been known for a much longer time. It is a common part of the language of glass mechanics at high temperatures, where the two notional processes that regulate mechanical behavior - shear-induced disordering and diffusional reordering or relaxation - are both active. Because the structural reordering process is thermally activated, at high temperatures it is entirely possible for deformation to be dominated by this diffusional process, which increases density and (presumably) structural order and strengthens the glass. This genuine structural strain hardening is most commonly appreciated during "stress undershoots", situations where a deforming glass is subjected to a sudden load drop, whereupon the diffusional reordering of the glass leads to a transient strengthening - that is, strain hardening - which occurs homogeneously (Fig. 14) 220].

Although the two processes of shear-induced disordering and diffusional reordering are both active at all temperatures, the general view has been that structural relaxation at low temperatures is slow. This allows the shear-induced disordering process (also sometimes thought of as a free volume-creation process) to dominate, leading to shear localization. Structural relaxation is still important because it affects the characteristic time scale for shear banding events [132] but under ordinary (uniaxial) loading conditions it cannot suppress shear localization entirely.

Recently, however, Wang and coworkers were able to achieve structural strain hardening in a Zr-based glass at ambient temperature 222. Those authors 
Figure 14: Data from strain-rate-drop experiments during homogeneous deformation in the non-Newtonian flow regime of $\mathrm{Zr}_{41.25} \mathrm{Ti}_{13.75} \mathrm{Cu}_{12.5} \mathrm{Ni}_{10} \mathrm{Be}_{22.5}$. At each strain rate the structure (and therefore the flow stress) reaches a steady state. When the strain rate is suddenly reduced there is a sudden drop in flow stress which is followed by structural ordering and strain hardening. Data from Ref. 221.

studied notched tensile specimens with a high stress triaxiality, and showed that the large hydrostatic tensile stresses promoted diffusional relaxation of the glass more than it did shear yielding. The result was a notch region that elongated significantly in tension without any shear localization, stabilized by the strain hardening process. They also explicitly demonstrated hardening in the specimen in the notch region with hardness tests as shown in Fig. 15. It appears that the glass was demonstrably hardened during plastic flow due to a local relaxation process promoted by the hydrostatic tensile component of stress.

This shows that some metallic glasses are capable of structural strain hardening under tensile loads, and most importantly, around notches and crack tips where stresses are concentrated and triaxiality is high. The implications of this mechanism for fracture have yet to be fully explored, but strain hardening around the crack tip is clearly highly desirable to increase fracture toughness. Wang and coworkers suggested that the ability of a glass to strain harden by stress-enhanced diffusional relaxation at a crack tip may be a determinant physical aspect separating brittle from tough glasses [222]. 


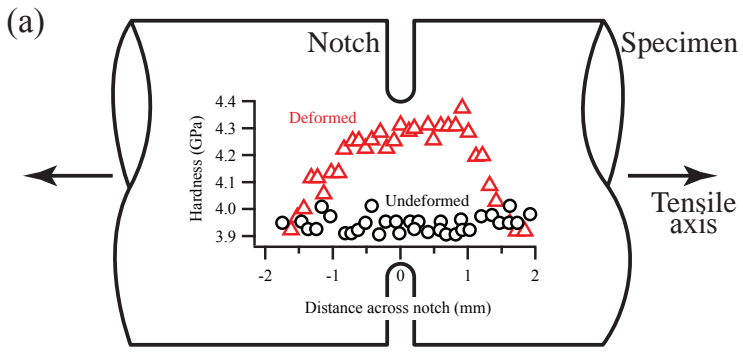

(b)
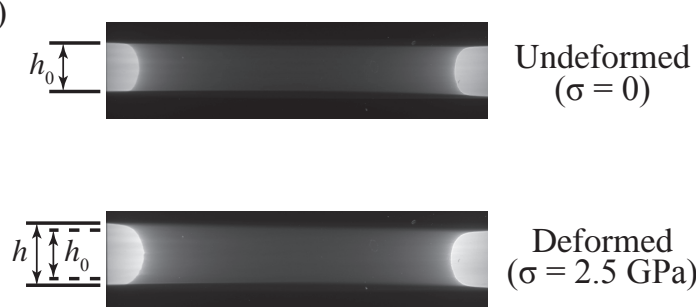

Figure 15: High aspect-ratio notches in tensile specimens promote strain hardening and plastic flow. (a) Hardness measured across the notched region of a specimen deformed to $7 \%$ plastic strain, compared to hardness of undeformed material. (Data from Ref. 222.) (b) In situ $\mathrm{x}$-ray radiographs of a notched specimen in the unloaded state (top) and loaded to $2.4 \mathrm{GPa}$ true stress (bottom). The extension of $h-h_{0}=77 \mu \mathrm{m}$ corresponds to an engineering strain of $22.5 \%$ in the notched region. 
Structural strain hardening at low temperatures is also suggested by studies

\section{2} a nominally elastic load with a hard indenter led to local hardening. After first loading the glass in the elastic range and demonstrating full strain recovery on unloading, they measured the yield point on a subsequent reload cycle as the 945 point where the first shear band was triggered and unrecoverable strain recorded. Using hundreds of data points in a statistical approach, they showed that elastic loading could cause a hardening by 20-30\%. STZ dynamics simulations as well as atomistic simulations 224, 225] provided support for the interpretation that around a stress concentration (such as the indenter tip, or perhaps a crack ${ }_{950}$ tip), local atomic-scale rearrangements are plausible before macroscopic yield is triggered and shear localization occurs.

Interestingly, cyclic loading appears to be particularly effective in producing these local atomic rearrangements with concomitant effects on the mechanical properties of the glass. For example, in the work cited above Packard and 955 coworkers also showed that cyclic loading in the elastic regime could progressively evoke strain hardening [225]. The implications of this for fatigue of metallic glasses would appear to be significant, but we are not aware of any studies of local hardening around fatigue crack tips as yet. The discovery of Ketov and coworkers, cited above, that thermal cycling to cryogenic temperatures causes structural rejuvenation, would appear to be a related effect [106].

It is worthwhile to note that the indentation studies and the notched tension experiments described above demonstrate structural strain hardening of metallic glasses for loading configurations with significant stress concentrations, and a similar comment could be made about the inhomogeneity of properties believed to be behind the cryogenic cycling effect. This underscores a need for greater understanding of STZs and related atomic structural changes in glasses under complex loading states in heterogeneous structures. 


\section{Directions for future research}

Throughout this review we have tried to emphasize connections between the

970

structure of metallic glasses and their mechanical behavior. From an experimental point of view, such connections are frustrated by the difficulty of obtaining precise, atomic- and nano-scale structural information about an amorphous material. In atomistic simulations we have perfectly precise information, but we lack quantitative descriptors of the key structural features that influence deformation behavior. In concluding this review we expand on these issues, identifying areas for research that are critically important for expanding our understanding of metallic glasses and which, on the basis of work to date, seem ripe for fruitful exploration.

- One critical question is the establishment of criteria for unambiguous identification of STZs - presuming, of course, that such defects are encoded in the structure of glasses. This is critical for testing the predictions of micromechanically-based constitutive laws and would open up the possibility of experimental verification of various metrics for identifying STZs. We note recent attempts to apply machine learning methods to automate the relation of structural measures to deformation activity [226]. This presents another promising area, particularly if the diagnostics derived in this way can be reverse-engineered to understand the origin of the connection between structure and defects.

- A related question is characterization of the spectrum of potential inelastic events that are available to the STZs in a given glass structure. Presumably these events range from mostly shear-like to mostly dilatational, with few being purely one or the other. A detailed, tensorial elaboration of the STZ spectrum at both the saddle point configurations and the final posttransition states would be of great interest. We see promise in using MD simulation and emerging structural characterization techniques (discussed below) to test predictions of constitutive models about the evolution of 
structure; direct comparisons between simulations and experiments are possible but will require new coarse-graining schemes and careful validation. Extending the analysis to different states of structural relaxation would provide great insight into some of the more complex issues associated with shear localization and fracture.

- It would also be desirable to develop quantifiable connections between constitutive theories and thermodynamic concepts. Experimentally this should be possible through calorimetry on macroscopic and microscopic specimens. Simulation methods that permit exploration of complex energy landscapes could in principle provide direct quantitative measures of configurational entropy by allowing the enumeration of the basins that comprise a meta-basin. In doing so, concepts like effective temperature become calculable and the predictions of theories that make reference to them directly testable.

- To date, studies of metallic glass deformation have emphasized uniaxial stress states. But studies of behavior and structural evolution under complex states of loading are urgently needed, for example to elucidate the processes of shear localization and fracture. The flow of shear momentum and free volume through the glass structure are clearly linked, but the details of this linkage and its role in controlling the balance of ordering and disordering are not well established. The elaboration of localization will require detailed and coupled understanding of these issues under multiaxial loading conditions. Much as studies of homogeneous deformation under uniaxial loading contributed to the development of a quantitative STZ theory, quantitative experimental work exploring multiaxial flow in the homogeneous regime could provide crucial input on these more complex issues.

- The fracture process itself is obviously of immense importance but as yet of limited understanding. But there is a relative paucity of information on fracture because in situ structural observations are difficult (due to the 
length scales and time scales involved) and because the heat released may significantly alter or destroy structural details that might reveal the evolution of the crack tip. This is an area where simulation may provide insights more readily than experiments. The relatively poorly-understood process of cavitation in glasses is a promising area for materials theory, because the inherent heterogeneity of the material on the smallest scales implies that the traditional separation between heterogeneous and homogeneous nucleation of cavities does not easily apply.

- Continuing advances in structural characterization are likely to improve our understanding of the structure of amorphous materials, including metallic glasses. For example, electron scattering techniques are being used to study atomic clusters in metallic glasses [227, 228] and nanometerscale structure and heterogeneity [181, 229]. More speculatively, the development of sources of spatially-coherent x-ray sources, already being used to probe the three-dimensional structure of nanocrystals, could ultimately provide the ability to resolve the atomic-scale structure of metallic glasses 230]. At this point, knowing position of every atom in the structure, experimentalists would face the same problem currently posed by atomic simulations: What to do with all that information?

- Even with existing techniques there are opportunities for significant advances in structural characterization. The importance of structural heterogeneity has been discussed above, so mapping of structure and properties on the nanometer scale is likely to be fruitful. Clever in situ experiments are also possible. For example, it has been shown that single shear bands evolve on time scales of seconds during deformation at cryogenic temperature, as opposed to millisecond time scales typical of deformation at ambient temperature [187]. This ability should make it feasible to study the atomic-scale structure around the advancing shear band using x-ray scattering, or the evolution of the macroscopic shear band structure using x-ray imaging (assuming the shear bands provide sufficient contrast). 
We close by noting that much of the enthusiasm for bulk metallic glasses began about two decades ago with major advances in alloy design which in turn led to innovations in metallic glass forming and processing science. Many

\section{Acknowledgements}

We gratefully acknowledge Katherine Jensen, Jun Ding, Evan Ma, and Chris Rycroft for providing calculations, data and illustrations used in several of the figures. TCH and CAS acknowledge the support of the U.S. National Science Foundation under Grants DMR-1408686 and CMMI-1332789, respectively. The images in Fig. 15(b) and data in Fig. 3 were taken at the Advanced Photon Source (APS) with the assistance of Mantong Zhao, Jiapeng Liu, Uday Vempati, Jon Almer, and Peter Kensei, under a research program supported by the National Science Foundation under Grant No. DMR-1408686. APS is a U.S. Department of Energy (DOE) Office of Science User Facility operated for the DOE Office of Science by Argonne National Laboratory under Contract No. DE-AC02-06CH11357.

\section{References}

[1] W. Klement, R. H. Willens, P. Duwez, Non-crystalline structures in solidified gold-silicon alloys, Nature 187 (1960) 869-870.

[2] H. W. Kui, A. L. Greer, D. Turnbull, Formation of bulk metallic-glass by fluxing, App. Phys. Lett. 45 (1984) 615-616. 
[3] A. Peker, W. Johnson, A highly processible metallic glass: $\mathrm{Zr}_{41.2} \mathrm{Ti}_{13.8} \mathrm{Cu}_{12.5} \mathrm{Ni}_{10.0} \mathrm{Be}_{22.5}$, Appl. Phys. Lett. 63 (1993) 2342-2344.

[4] A. Inoue, Stabilization of metallic supercooled liquid and bulk amorphous alloys, Acta Mater. 48 (2000) 279-306.

[5] C. A. Schuh, T. C. Hufnagel, U. Ramamurty, Mechanical properties of

[12] M. L. Falk, C. E. Maloney, Simulating the mechanical response of amorphous solids using atomistic methods, European Physical Journal B 75 (4) (2010) 405-413. doi:\{10.1140/epjb/e2010-00157-7\}. 
[13] D. Rodney, A. Tanguy, D. Vandembroucq, Modeling the mechanics of amorphous solids at different length scale and time scale, Modelling and Simulation in Materials Science and Engineering 19 (8) (2011) 083001. doi: $\{10.1088 / 0965-0393 / 19 / 8 / 083001\}$.

[14] E. Ma, Tuning order in disorder, Nature Materials 14 (6) (2015) 547-552.

[15] J. Ding, Y. Q. Cheng, E. Ma, Full icosahedra dominate local order in $\mathrm{Cu}_{64} \mathrm{Zr}_{34}$ metallic glass and supercooled liquid, Acta Materialia 69 (2014) 343-354.

[16] F. Spaepen, Condensed-matter science - Five-fold symmetry in liquids, Nature 408 (6814) (2000) 781-782.

[17] Y. Q. Cheng, E. Ma, H. W. Sheng, Atomic Level Structure in Multicomponent Bulk Metallic Glass, Physical Review Letters 102 (24) (2009) 245501. doi:\{10.1103/PhysRevLett.102.245501\},

[18] Y. Q. Cheng, H. W. Sheng, E. Ma, Relationship between structure, dynamics, and mechanical properties in metallic glass-forming alloys, Physical Review B 78 (1) (2008) 014207. doi:10.1103/PhysRevB.78.014207

[19] Y. Q. Cheng, A. J. Cao, E. Ma, Correlation between the elastic modulus and the intrinsic plastic behavior of metallic glasses: The roles of atomic configuration and alloy composition, Acta Mater. 57 (11) (2009) 32533267. doi:10.1016/j.actamat.2009.03.027.

[20] Y. Q. Cheng, E. Ma, H. W. Sheng, Alloying strongly influences the structure, dynamics, and glass forming ability of metallic supercooled liquids, Appl. Phys. Lett. 93 (11) (2008) 111913. doi:10.1063/1.2987727.

[21] Y. Q. Cheng, A. J. Cao, H. W. Sheng, E. Ma, Local order influences initiation of plastic flow in metallic glass: Effects of alloy composition and sample cooling history, Acta Mater. 56 (18) (2008) 5263-5275. doi: 10.1016/j.actamat.2008.07.011 
[22] J. Ding, S. Patinet, M. L. Falk, Y. Cheng, E. Ma, Soft spots and their structural signature in a metallic glass, Proceedings of the National Academy of Sciences of the United States of America 111 (39) (2014) 14052-14056.

[23] F. H. Stillinger, P. G. Debenedetti, Glass transition thermodynamics and kinetics, Annu. Rev. Condens. Matter Phys. 4 (1) (2013) 263-285.

[24] M. D. Ediger, Spatially heterogeneous dynamics in supercooled liquids, Annual Review of Physical Chemistry 51 (1) (2000) 99-128.

[25] S. Karmakar, C. Dasgupta, S. Sastry, J. S. Langer, Growing length scales and their relation to timescales in glass-forming liquids, Annual Review of Condensed Matter Physics 5 (2014) 255-284. doi:10.1146/ annurev-conmatphys-031113-133848.

[26] D. B. Miracle, A structural model for metallic glasses, Nature Mater. 3 (10) (2004) 697-702.

[27] D. B. Miracle, A physical model for metallic glass structures: An inn troduction and update, JOM 64 (7) (2012) 846-855. doi:10.1007/ s11837-012-0359-4

[28] I. C. Noyan, J. B. Cohen, Residual Stress: Measurement by Diffraction and Interpretation, Springer-Verlag, New York, 1987.

[29] H. F. Poulsen, J. A. Wert, J. Neuefeind, V. Honkimaki, M. Daymond, Measuring strain distributions in amorphous materials, Nature Mater. 4 (2005) 33-36.

[30] M. Pick, R. Lovell, A. Windle, Detection of elastic strain in an amorphous polymer by x-ray scattering, Nature 281 (5733) (1979) 658-659.

[31] M. Stoica, J. Das, J. Bednarcik, G. Wang, G. Vaughan, W. H. Wang, J. Eckert, Mechanical response of metallic glasses: Insights from in-situ high energy x-ray diffraction, JOM 62 (2010) 76-82. 
[32] T. C. Hufnagel, U. K. Vempati, J. Almer, Crack-tip strain field mapping and the toughness of metallic glasses, PLoS ONE 8 (12) (2013) e83289.

[33] T. C. Hufnagel, R. T. Ott, J. Almer, Structural aspects of elastic deformation of a metallic glass, Phys. Rev. B 73 (2006) 064204.

[

[34] J. Das, M. Bostrom, N. Mattern, A. Kvick, A. R. Yavari, A. L. Greer, J. Eckert, Plasticity in bulk metallic glasses investigated via the strain distribution, Physical Review B 76 (2007) 092203. doi:DOI10.1103/ PhysRevB.76.092203.

[35] F. O. Mear, G. Vaughan, A. R. Yavari, A. L. Greer, Residual-stress distribution in shot-peened metallic-glass plate, Philosophical Magazine Letters 88 (2008) 757-766. doi:DOI10.1080/09500830802235776.

[36] S. Michalik, J. Michalikova, M. Pavlovic, P. Sovak, H. P. Liermann, M. Miglierini, Structural modifications of swift-ion-bombarded metallic glasses studied by high-energy X-ray synchrotron radiation, Acta Materialia 80 (2014) 309-316.

[37] A. R. Yavari, A. L. Moulec, A. Inoue, N. Nishiyama, N. Lupu, E. Matsubara, W. J. Botta, G. Vaughan, M. D. Michiel, A. Kvick, Excess free volume in metallic glasses measured by x-ray diffraction, Acta Mater. 53 (2005) 1611-1619.

[38] N. Mattern, J. Bednarcik, S. Pauly, G. Wang, J. Das, J. Eckert, Structural evolution of $\mathrm{Cu}-\mathrm{Zr}$ metallic glasses under tension, Acta Materialia 57 (14) (2009) 4133-4139.

[39] M. Stoica, S. Scudino, J. Bednarčik, I. Kaban, J. Eckert, FeCoSiBNbCu bulk metallic glass with large compressive deformability studied by timeresolved synchrotron x-ray diffraction, Journal of Applied Physics 115 (2014) 053520.

[40] S. Sato, H. Suzuki, T. Shobu, M. Imafuku, Y. Tsuchiya, K. Wagatsuma, H. Kato, A. D. Setyawan, J. Saida, Atomic-scale characterization of elas- 
tic deformation of Zr-based metallic glass under tensile stress, Materials Transactions 51 (8) (2010) 1381-1385.

[41] X. D. Wang, J. Bednarcik, K. Saksl, H. Franz, Q. P. Cao, J. Z. Jiang, Tensile behavior of bulk metallic glasses by in situ x-ray diffraction, Applied Physics Letters 91 (8) (2007) 081913.

[42] M. Stoica, J. Das, J. Bednarcik, H. Franz, N. Mattern, W. H. Wang, J. Eckert, Strain distribution in $\mathrm{Zr}_{64.13} \mathrm{Cu}_{15.75} \mathrm{Ni}_{10.12} \mathrm{Al}_{10}$ bulk metallic glass investigated by in situ tensile tests under synchrotron radiation, Journal of Applied Physics 104 (1) (2008) 013522.

[43] G. Wang, N. Mattern, S. Pauly, J. Bednarcik, J. Eckert, Atomic structure evolution in bulk metallic glass under compressive stress, Applied Physics Letters 95 (25) (2009) 251906.

[44] U. K. Vempati, J. Almer, T. C. Hufnagel, upublished.

[45] X. D. Wang, H. B. Lou, J. Bednarcik, H. Franz, H. W. Sheng, Q. P. Cao, J. Z. Jiang, Structural evolution in bulk metallic glass under hightemperature tension, Applied Physics Letters 102 (5) (2013) 051909.

[46] W. Dmowski, T. Egami, Observation of structural anisotropy in metallic glasses induced by mechanical deformation, Journal of Materials Research 22 (02) (2011) 412-418.

[47] U. K. Vempati, P. K. Valavala, M. L. Falk, J. Almer, T. C. Hufnagel, Length-scale dependence of elastic strain from scattering measurements in metallic glasses, Phys. Rev. B 85 (2012) 214201.

[48] D. Weaire, M. F. Ashby, J. Logan, M. J. Weins, On the use of pair potentials to calculate the properties of amorphous metals, Acta Metall. 19 (1971) 779-788.

[49] Y. Suzuki, T. Egami, Shear deformation of glassy metals: Breakdown 1220 of Cauchy relationship and anelasticity, J. Non-Cryst. Solids 75 (1985) 361-366. 
[50] A. Lemaitre, C. Maloney, Sum rules for the quasi-static and visco-elastic response of disordered solids at zero temperature, Journal of Statistical Physics 123 (2) (2006) 415-453. doi:\{10.1007/s10955-005-9015-5\}.

[52] K. Yoshimoto, T. S. Jain, K. V. Workum, P. F. Nealey, J. J. de Pablo, Mechanical heterogeneities in model polymer glasses at small length scales,

[51] K. Van Workum, J. J. de Pablo, Local elastic constants in thin films of an fcc crystal, Phys. Rev. E 67 (3) (2003) 031601. doi:10.1103/PhysRevE. 67.031601 Phys. Rev. Lett. 93 (17) (2004) 175501. doi:10.1103/PhysRevLett.93. 175501 .

[53] K. Maeda, S. Takeuchi, Atomistic processes of plastic-deformation in a model amorphous metal, Phil. Mag. A 44 (1981) 643-651.

[54] M. Tsamados, A. Tanguy, C. Goldenberg, J.-L. Barrat, Local elasticity map and plasticity in a model Lennard-Jones glass, Physical Review E 80 (2009) 026112. doi:\{10.1103/PhysRevE.80.026112\}.

[55] F. Leonforte, R. Boissière, A. Tanguy, J. Wittmer, J. L. Barrat, Continuum limit of amorphous elastic bodies. III. Three-dimensional systems, Physical Review B 72 (22) (2005) 224206.

[56] F. Leonforte, A. Tanguy, J. P. Wittmer, J. L. Barrat, Inhomogeneous elastic response of silica glass, Phys. Rev. Lett. 97 (5) (2006) 055501. doi:10.1103/PhysRevLett.97.055501.

[57] C. Goldenberg, I. Goldhirsch, Force chains, microelasticity, and macroelasticity, Phys. Rev. Lett. 89 (8) (2002) 084302. doi:10.1103/PhysRevLett. 89.084302.

[58] A. Heuer, Exploring the potential energy landscape of glass-forming systems: From inherent structures via metabasins to macroscopic trans口 port, J. Phys.-Condes. Matter 20 (37) (2008) 373101. doi:10.1088/ 0953-8984/20/37/373101. 
[59] P. G. Debenedetti, F. H. Stillinger, Supercooled liquids and the glass transition, Nature 410 (6825) (2001) 259-267.

[60] D. Rodney, C. Schuh, Distribution of thermally activated plastic events in a flowing glass, Physical Review Letters 102 (23) (2009) 235503-4.

[61] H.-B. Yu, W.-H. Wang, K. Samwer, The beta relaxation in metal1255 _ lic glasses: an overview, Mater. Today 16 (5) (2013) 183-191. doi: $10.1016 / j$.mattod.2013.05.002

[62] G. P. Johari, M. Goldstein, Molecular mobility in simple glasses, J. Phys. Chem. 74 (9) (1970) 2034-2035.

[63] J. M. Pelletier, B. Van de Moortele, I. R. Lu, Viscoelasticity and viscosity of pd-ni-cu-p bulk metallic glasses, Mater. Sci. Eng. A-Struct. Mater. Prop. Microstruct. Process. 336 (1-2) (2002) 190-195.

[64] D. L. Malandro, D. J. Lacks, Relationships of shear-induced changes in the potential energy landscape to the mechanical properties of ductile glasses, J. Chem. Phys. 110 (9) (1999) 4593-4601.

[65] D. J. Lacks, M. J. Osborne, Energy landscape picture of overaging and rejuvenation in a sheared glass, Physical Review Letters 93 (25) (200) 255501.

[66] Y. Fan, T. Iwashita, T. Egami, How thermally activated deformation starts in metallic glass, Nature Communications 5 (2014) 5083-7.

1270 [67] A. S. Argon, H. Y. Kuo, Plastic-flow in a disordered bubble raft (an analog of a metallic glass), Materials Science and Engineering 39 (1) (1979) 101109.

[68] A. S. Argon, L. T. Shi, Analysis of plastic-flow in an amorphous soap bubble raft by the use of an inter-bubble potential, Philos. Mag. A 46 (2) (1982) 275-294. 
[69] T. Tomida, T. Egami, Molecular-dynamics study of structural anisotropy and anelasticity in metallic glasses, Physical Review B 48 (5) (1993) 30483057. doi:\{10.1103/PhysRevB.48.3048\}.

[70] Z. Wang, P. Wen, L. S. Huo, H. Y. Bai, W. H. Wang, Signature of viscous flow units in apparent elastic regime of metallic glasses, Applied Physics Letters 101 (12) (2012) 121906. doi:\{10.1063/1.4753813\}.

[71] J. D. Ju, D. Jang, A. Nwankpa, M. Atzmon, An atomically quantized hierarchy of shear transformation zones in a metallic glass, Journal of Applied Physics 109 (5) (2011) 053522. doi:\{10.1063/1.3552300\}

[76] A. Tanguy, F. Leonforte, J. L. Barrat, Plastic response of a 2D LennardJones amorphous solid: Detailed analysis of the local rearrangements at very slow strain rate, European Physical Journal E 20 (3) (2006) 355-364. doi:\{10.1140/epje/i2006-10024-2\}. 
[77] P. Cao, H. S. Park, X. Lin, Strain-rate and temperature-driven transition in the shear transformation zone for two-dimensional amorphous solids, Physical Review E 88 (4) (2013) 042404. doi:\{10.1103/PhysRevE.88. 042404\}.

[78] E. R. Homer, D. Rodney, C. A. Schuh, Kinetic Monte Carlo study of activated states and correlated shear-transformation-zone activity during the deformation of an amorphous metal, Physical Review B 81 (6) (2010) 064204. doi:\{10.1103/PhysRevB.81.064204\}.

[79] D. Pan, A. Inoue, T. Sakurai, M. W. Chen, Experimental characterization of shear transformation zones for plastic flow of bulk metallic glasses, Proceedings of the National Academy of Sciences of the United States of America 105 (39) (2008) 14769-14772. doi:\{10.1073/pnas. $0806051105\}$

[80] D. Pan, Y. Yokoyama, T. Fujita, Y. H. Liu, S. Kohara, A. Inoue, M. W. Chen, Correlation between structural relaxation and shear transformation zone volume of a bulk metallic glass, Applied Physics Letters 95 (14) (2009) 141909. doi:\{10.1063/1.3246151\}.

1320

口

[81] F. Jiang, M. Q. Jiang, H. F. Wang, Y. L. Zhao, L. He, J. Sun, Shear transformation zone volume determining ductile-brittle transition of bulk metallic glasses, Acta Materialia 59 (5) (2011) 2057-2068. doi:\{10.1016/ j.actamat.2010.12.006\}.

[82] I.-C. Choi, Y. Zhao, Y.-J. Kim, B.-G. Yoo, J.-Y. Suh, U. Ramamurty, J.-I. Jang, Indentation size effect and shear transformation zone size in a bulk metallic glass in two different structural states, Acta Materialia 60 (19) (2012) 6862-6868. doi:\{10.1016/j .actamat.2012.08.061\}.

[83] Y. Zhao, I.-C. Choi, M.-Y. Seok, M.-H. Kim, D.-H. Kim, U. Ramamurty, J.-Y. Suh, J.-I. Jang, Effect of hydrogen on the yielding behavior and shear transformation zone volume in metallic glass ribbons, Acta Materialia 78 (2014) 213-221. doi:\{10.1016/j.actamat.2014.06.046\}. 
[84] P. Schall, D. A. Weitz, F. Spaepen, Structural rearrangements that govern flow in colloidal glasses, Science 318 (5858) (2007) 1895-1899.

[85] K. E. Jensen, D. A. Weitz, F. Spaepen, Local shear transformations in deformed and quiescent hard-sphere colloidal glasses, Physical Review E 90 (4) (2014) 042305.

[86] A. Argon, Plastic deformation in metallic glasses, Acta Metall. 27 (1979) $47-58$.

[87] F. Spaepen, A microscopic mechanism for steady state inhomogeneous flow in metallic glasses, Acta Metall. 25 (1977) 407-415.

[88] C. Maloney, A. Lemaitre, Subextensive scaling in the athermal, quasistatic limit of amorphous matter in plastic shear flow, Physical Review Letters 93 (1) (2004) 016001. doi:\{10.1103/PhysRevLett.93.016001\}.

[89] C. Maloney, A. Lemaître, Universal breakdown of elasticity at the onset of material failure, Physical Review Letters 93 (19) (2004) 195501. doi: $\{10.1103 /$ PhysRevLett.93.195501\}.

[90] C. E. Maloney, A. Lemaître, Amorphous systems in athermal, quasistatic shear, Physical Review E 74 (2006) 016118. doi:\{10.1103/PhysRevE. $74.016118\}$

[91] A. Lemaitre, C. Caroli, Rate-dependent avalanche size in athermally sheared amorphous solids, Phys. Rev. Lett. 103 (6) (2009) 065501. doi: 10.1103/PhysRevLett.103.065501

[92] A. Lemaître, C. Caroli, Plastic response of a two-dimensional amorphous solid to quasistatic shear: Transverse particle diffusion and phenomenology of dissipative events, Physical Review E 76 (2007) 036104. doi:\{10.1103/PhysRevE.76.036104\}.

[93] K. M. Salerno, C. E. Maloney, M. O. Robbins, Avalanches in strained amorphous solids: does inertia destroy critical behavior?, Physical Re- 
view Letters 109 (10) (2012) 105703. doi:\{10.1103/PhysRevLett.109. $105703\}$.

[94] B. B. Laird, H. R. Schober, Localized low-frequency vibrational-modes in a simple-model glass, Physical Review Letters 66 (5) (1991) 636-639. doi:\{10.1103/PhysRevLett.66.636\}.

[95] H. R. Schober, B. B. Laird, Localized low-frequency vibrational-modes 1365 in glasses, Physical Review B 44 (13) (1991) 6746-6754. doi:\{10.1103/ PhysRevB.44.6746\}.

[96] H. R. Schober, C. Oligschleger, B. B. Laird, Low-frequency vibrations and relaxations in glasses, Journal of Non-Crystalline Solids 156 (1993) 965-968. doi: $\{10.1016 / 0022-3093(93) 90106-8\}$.

1. glass, Physical Review B 53 (17) (1996) 11469-11480. doi:\{10.1103/ PhysRevB.53.11469\}.

[98] M. L. Manning, A. J. Liu, Vibrational modes identify soft spots in a sheared disordered packing, Physical Review Letters 107 (10) (2011) 108302. doi:\{10.1103/PhysRevLett.107.108302\}

[99] S. S. Schoenholz, A. J. Liu, R. A. Riggleman, J. Rottler, Understanding plastic deformation in thermal glasses from single-soft-spot dynam-

口 ics, Physical Review X 4 (3) (2014) 031014. doi:\{10.1103/PhysRcvX.4. $031014\}$.

[100] J. Rottler, S. S. Schoenholz, A. J. Liu, Predicting plasticity with soft vibrational modes: From dislocations to glasses, Physical Review E 89 (4) (2014) 042304. doi:\{10.1103/PhysRevE.89.042304\}.

[101] T. Ichitsubo, S. Hosokawa, K. Matsuda, E. Matsubara, N. Nishiyama, S. Tsutsui, A. Baron, Nanoscale elastic inhomogeneity of a Pd-based metallic glass: Sound velocity from ultrasonic and inelastic x-ray scattering experiments, Physical Review B 76 (14) (2007) 140201. 
[102] H. Wagner, D. Bedorf, S. Kuechemann, M. Schwabe, B. Zhang, W. Arnold, K. Samwer, Local elastic properties of a metallic glass, Nature Materials 10 (6) (2011) 439-442.

1390

[103] C. E. Packard, O. Franke, E. R. Homer, C. A. Schuh, Nanoscale strength distribution in amorphous versus crystalline metals, J. Mater. Res. 25 (12) (2010) 2251-2263. doi:10.1557/JMR.2010.0299

[104] Y. H. Liu, D. Wang, K. Nakajima, W. Zhang, A. Hirata, T. Nishi, A. Inoue, M. W. Chen, Characterization of nanoscale mechanical heterogeneity in a metallic glass by dynamic force microscopy, Physical Review Letters 106 (12) (2011) 125504.

[105] Y. Luo, Q.-K. Li, M. Li, Mechanical anisotropy at the nanoscale in amorphous solids, Journal of Applied Physics 117 (4) (2015) 044301.

[106] S. V. Ketov, Y. H. Sun, S. Nachum, Z. Lu, A. Checchi, A. R. Beraldin, H. Y. Bai, W. H. Wang, D. V. Louzguine-Luzgin, M. A. Carpenter, A. L. Greer, Greer, Rejuvenation of metallic glasses by non-affine thermal strain, Nature 524 (2015) 200-203.

[107] W. Dmowski, T. Iwashita, C.-P. Chuang, J. Almer, T. Egami, Elastic heterogeneity in metallic glasses, Physical Review Letters 105 (20) (2010) 205502.

[108] P. S. Steif, F. Spaepen, J. W. Hutchinson, Strain localization in amorphous metals, Acta Metall. 30 (1982) 447-455.

[109] R. Huang, Z. Suo, J. H. Prevost, W. D. Nix, Inhomogeneous deformation in metallic glasses, J. Mech. Phys. Sol. 50 (2002) 1011-1027. 
[111] D. Turnbull, M. H. Cohen, Free-volume model of amorphous phase - glass transition, Journal of Chemical Physics 34 (1) (1961) 120-\&. doi:\{10. $1063 / 1.1731549\}$.

[112] D. Turnbull, M. H. Cohen, On free-volume model of liquid-glass transition, Journal of Chemical Physics 52 (6) (1970) 3038-\&. doi:\{10.1063/1. $1673434\}$.

[113] L. Anand, C. Su, A theory for amorphous viscoplastic materials undergoing finite deformation, with application to metallic glasses, J. Mech. Phys. Sol. 53 (2005) 1362-1396.

[114] C. Su, L. Anand, Plane strain indentation of a Zr-based metallic glass: Experiments and numerical simulation, Acta Mater. 54 (2006) 179-189.

[115] L. Anand, C. Su, A constitutive theory for metallic glasses at high 1425 homologous temperatures, Acta Materialia 55 (11) (2007) 3735-3747. doi: $\{10.1016 / j$. actamat.2007.02.020\}.

[116] H. H. Ruan, L. C. Zhang, J. Lu, A new constitutive model for shear banding instability in metallic glass, International Journal of Solids and

1. Structures 48 (21) (2011) 3112-3127. doi:\{10.1016/j.ijsolstr.2011. $07.004\}$.

[117] W. L. Johnson, J. Lu, M. D. Demetriou, Deformation and flow in bulk metallic glasses and deeply undercooled glass forming liquids-a self consistent dynamic free volume model, Intermetallics 10 (2002) 1039-1046.

[118] M. L. Falk, J. S. Langer, Deformation and failure of amorphous, solidlike materials, Annual Review of Condensed Matter Physics 2 (2011) 353-373. doi:\{10.1146/annurev-conmatphys-062910-140452\},

[119] M. Falk, J. Langer, Dynamics of viscoplastic deformation in amorphous solids, Physical Review E 57 (6) (1998) 7192-7205. doi:\{10.1103/ PhysRevE.57.7192\}. 
1465

[120] E. Bouchbinder, J. S. Langer, Nonequilibrium thermodynamics of driven amorphous materials. I. Internal degrees of freedom and volume deforma-

[ tion, Physical Review E 80 (3) (2009) 031131. doi:\{10.1103/PhysRevE. $80.031131\}$.

[121] K. Kamrin, E. Bouchbinder, Two-temperature continuum thermomechanics of deforming amorphous solids, Journal of the Mechanics and Physics of Solids 73 (2014) 269-288. doi:\{10.1016/j.jmps.2014.09.009\}.

[122] Y. Shi, M. B. Katz, H. Li, M. L. Falk, Evaluation of the disorder temperature and free-volume formalisms via simulations of shear banding in amorphous solids, Physical Review Letters 98 (2007) 185505.

口

[124] C. H. Rycroft, Y. Sui, E. Bouchbinder, An eulerian projection method 1455

[126] S.-C. Lee, C.-M. Lee, J.-C. Lee, H.-J. Kim, Y. Shibutani, E. Fleury, M. L. Falk, Structural disordering process of an amorphous alloy driven by the elastostatic compression at room temperature, Applied Physics Letters 92 (15) (2008) 151906. doi:\{10.1063/1.2908218\}.

[127] K.-W. Park, C.-M. Lee, M. Wakeda, Y. Shibutani, M. L. Falk, J.-C. Lee, Elastostatically induced structural disordering in amorphous alloys, Acta 
Materialia 56 (19) (2008) 5440-5450. doi:\{10.1016/j.actamat.2008. $07.033\}$.

[128] M. Falk, J. Langer, L. Pechenik, Thermal effects in the sheartransformation-zone theory of amorphous plasticity: Comparisons to

口 metallic glass data, Physical Review E 70 (2004) 011507. doi:\{10.1103/ PhysRevE.70.011507\}.

[129] N. Perchikov, E. Bouchbinder, Variable-amplitude oscillatory shear response of amorphous materials, Physical Review E 89 (6) (2014) 062307. doi:\{10.1103/PhysRevE.89.062307\}.

[130] E. G. Daub, D. Klaumuenzer, J. F. Loeffler, Effective temperature dynamics of shear bands in metallic glasses, Physical Review E 90 (6) (2014) 062405. doi:\{10.1103/PhysRevE.90.062405\},

[131] C. A. Pampillo, Flow and fracture in amorphous alloys, J. Mat. Sci. 10 (1975) 1194-1227.

[132] Maass, Robert and Loeffler, Joerg F, Shear-band dynamics in metallic glasses, Advanced Functional Materials 25 (16) (2015) 2353-2368.

[133] Y. Q. Cheng, E. Ma, Intrinsic shear strength of metallic glass, Acta Materialia 59 (4) (2011) 1800-1807. doi:\{10.1016/j .actamat.2010.11.046\}

[134] Y. Shi, M. L. Falk, Atomic-scale simulations of strain localization in threedimensional model amorphous solids, Phys. Rev. B 73 (21) (2006) 10. doi:10.1103/PhysRevB.73.214201.

[135] N. P. Bailey and J. Schiøtz and K. W. Jacobsen, Atomistic simulation of the shear-band deformation mechanism in $\mathrm{Mg}-\mathrm{Cu}$ metallic glasses, Phys. Rev. B 73 (2006) 064108.

[136] Y. Shi, M. L. Falk, Stress-induced structural transformation and shear banding during simulated nanoindentation of a metallic glass, Acta Materialia 55 (13) (2007) 4317-4324. doi:DOI10.1016/j.actamat.2007.03. 029 . 
[137] A. J. Cao, Y. Q. Cheng, E. Ma, Structural processes that initiate shear localization in metallic glass, Acta Materialia 57 (17) (2009) 5146-5155. doi: $\{10.1016 / j$.actamat.2009.07.016\}

[138] F. Shimizu, S. Ogata, J. Li, Yield point of metallic glass, Acta Materialia 54 (16) (2006) 4293-4298. doi:\{10.1016/j.actamat.2006.05.024\}.

[139] W. J. Wright, M. W. Samale, T. C. Hufnagel, M. M. LeBlanc, J. N. Florando, Studies of shear band velocity using spatially and temporally resolved measurements of strain during quasistatic compression of a bulk metallic glass, Acta Materialia 57 (16) (2009) 4639-4648.

[140] S. X. Song, T. G. Nieh, Flow serration and shear-band viscosity during inhomogeneous deformation of a Zr-based bulk metallic glass, Intermetallics 17 (9) (2009) 762-767. doi:\{10.1016/j.intermet.2009.03.005\}.

[141] S. X. Song, T. G. Nieh, Direct measurements of shear band propagation in metallic glasses - An overview, Intermetallics 19 (12) (2011) 1968-1977. doi: $\{10.1016 / j$. intermet.2011.06.018\}.

[142] W. J. Wright, R. R. Byer, X. Gu, High-speed imaging of a bulk metallic glass during uniaxial compression, Applied Physics Letters 102 (2013) 241920. doi: $\{10.1063 / 1.4811744\}$.

[143] S. Ketov, V, D. Louzguine-Luzgin, V, Localized shear deformation and softening of bulk metallic glass: Stress or temperature driven?, Scientific Reports 3 (2013) 2798. doi:\{10.1038/srep02798\}.

[144] S. K. Slaughter, F. Kertis, E. Deda, X. Gu, W. J. Wright, T. C. Hufnagel, Shear bands in metallic glasses are not necessarily hot, APL Materials 2 (2014) 096110. doi:\{10.1063/1.4895605\}.

[145] T. C. Hufnagel, P. El-Deiry, R. P. Vinci, Development of shear band structure during deformation of a $\mathrm{Zr}_{57} \mathrm{Ti}_{5} \mathrm{Cu}_{20} \mathrm{Ni}_{8} \mathrm{Al}_{10}$ bulk metallic glass, Scripta Mat. 12 (2000) 1071-1074. 
[146] K. M. Flores, R. H. Dauskardt, Enhanced toughness due to stable crack tip damage zones in bulk metallic glass, Scripta Metall. 41 (1999) 937-43.

[147] S. X. Song, X. L. Wang, T. G. Nieh, Capturing shear band propagation in a Zr-based metallic glass using a high-speed camera, Scripta Materialia 62 (11) (2010) 847-850. doi:\{10.1016/j.scriptamat.2010.02.017\}.

[148] Z. Han, Y. Li, Cooperative shear and catastrophic fracture of bulk metallic glasses from a shear-band instability perspective, Journal of Materials Research 24 (12) (2009) 3620-3627. doi:\{10.1557/JMR.2009.0442\}.

[149] R. T. Qu, Z. Q. Liu, G. Wang, Z. F. Zhang, Progressive shear band propagation in metallic glasses under compression, Acta Materialia 91 (2015) 19-33.

[150] P. Guan, M. Chen, T. Egami, Stress-temperature scaling for steady-state flow in metallic glasses, Physical Review Letters 104 (20) (2010) 205701. doi:10.1103/PhysRevLett.104.205701

[151] J. J. Lewandowski, A. L. Greer, Temperature rise at shear bands in metallic glasses, Nature Mater. 5 (2006) 15-18.

[152] Y. Cheng, Z. Han, Y. Li, E. Ma, Cold versus hot shear banding in bulk metallic glass, Physical Review B 80 (13) (2009) 134115.

[153] D. B. Miracle, A. Concustell, Y. Zhang, A. R. Yavari, A. L. Greer, Shear bands in metallic glasses: Size effects on thermal profiles, Acta Materialia 59 (7) (2011) 2831-2840. doi:\{10.1016/j.actamat.2011.01.022\},

[154] Z. Zhang, J. Eckert, L. Schultz, Difference in compressive and tensile fracture mechanisms of Zr59CU20Al10Ni8Ti3 bulk metallic glass, Acta Materialia 51 (4) (2003) 1167-1179. doi:\{10.1016/S1359-6454(02) 00521-9\}.

[155] E. Bouchaud, D. Boivin, J.-L. Pouchou, D. Bonamy, B. Poon, G. Ravichandran, Fracture through cavitation in a metallic glass, EPL 83 (6) (2008) 66006. doi:\{10.1209/0295-5075/83/66006\}. 
[162] C. A. Volkert, A. Donohue, F. Spaepen, Effect of sample size on deformation in amorphous metals, Journal Of Applied Physics 103 (8) (2008) 083539 .

[163] B. E. Schuster, Q. Wei, T. C. Hufnagel, K. T. Ramesh, Size-independent 1575

[156] P. Guan, S. Lu, M. J. B. Spector, P. Valavala, M. L. Falk, Cavitation in amorphous solids, Phys. Rev. Lett. 110 (2013) 185502.

[157] P. Murali, T. F. Guo, Y. W. Zhang, R. Narasimhan, Y. Li, H. J. Gao, Atomic Scale Fluctuations Govern Brittle Fracture and Cavitation Behavior in Metallic Glasses, Physical Review Letters 107 (21) (2011) 215501. doi:\{10.1103/PhysRevLett.107.215501\}.

[158] A. Vinogradov, A. Lazarev, D. V. Louzguine-Luzgin, Y. Yokoyama, S. Li, A. R. Yavari, A. Inoue, Propagation of shear bands in metallic glasses and transition from serrated to non-serrated plastic flow at low temperatures, Acta Materialia 58 (20) (2010) 6736-6743. doi:\{10.1016/j.actamat. $2010.08 .039\}$.

[159] D. Klaumuenzer, A. Lazarev, R. Maass, F. H. D. Torre, A. Vinogradov, J. F. Loeffler, Probing shear-band initiation in metallic glasses, Physical 口 $107.185502\}$

[160] H. Guo, P. F. Yan, Y. B. Wang, J. Tan, Z. F. Zhang, M. L. Sui, E. Ma, Tensile ductility and necking of metallic glass, Nature Materials 6 (10) (2007) 735-739.

[161] Z. W. Shan, J. Li, Y. Q. Cheng, A. M. Minor, S. A. S. Asif, O. L. Warren, E. Ma, Plastic flow and failure resistance of metallic glass: Insight from in situ compression of nanopillars, Physical Review B 77 (15) (2008) 155419. strength and deformation mode in compression of a Pd-based metallic glass, Acta Materialia 56 (18) (2008) 5091-5100. 
[164] A. Dubach, R. Raghavan, J. F. Löffler, J. Michler, U. RAMAMURTY, Micropillar compression studies on a bulk metallic glass in different structural states, Scripta Materialia 60 (7) (2009) 567-570.

[165] J. R. Greer, J. T. M. De Hosson, Plasticity in small-sized metallic systems: Intrinsic versus extrinsic size effect, Progress in Materials Science 56 (6) (2011) 654-724.

[166] D. Tönnies, R. Maass, C. A. Volkert, Room temperature homogeneous ductility of micrometer-sized metallic glass, Advanced Materials 26 (32) (2014) 5715-5721.

[167] Y. Zhang, A. L. Greer, Thickness of shear bands in metallic glasses, Appl. Phys. Lett. 89 (7) (2006) 071907. doi:10.1063/1.2336598

[168] Y. M. Chen, T. Ohkubo, T. Mukai, K. Hono, Structure of shear bands in $\mathrm{Pd}_{40} \mathrm{Ni}_{40} \mathrm{P}_{20}$ bulk metallic glass, Journal of Materials Research 24 (1) (2009) 1-9. doi:10.1557/JMR. 2009.0001.

[169] A. C. Y. Liu, D. M. Paganin, L. Bourgeois, P. N. H. Nakashima, R. T. Ott, M. J. Kramer, Quantitative microscopic measurement of void distribution in shear bands in $\mathrm{Zr}_{66.7} \mathrm{Cu}_{33.3}$ metallic glass, Physical Review B 84 (2011) 094201. doi:10.1103/PhysRevB.84.094201.

[170] H. Roesner, M. Peterlechner, C. Kuebel, V. Schmidt, G. Wilde, Density changes in shear bands of a metallic glass determined by correlative analytical transmission electron microscopy, Ultramicroscopy 142 (2014) 1-9. doi:10.1016/j.ultramic.2014.03.006

[171] R. W. Cahn, N. A. Pratten, M. G. Scott, H. R. Sinning, L. Leonardsson, Studies of relaxation of metallic glasses by dilatometry and density measurements, Mat. Res. Soc. Symp. Proc. 28 (1984) 241-252.

[172] H. S. Chen, Stored energy in a cold-rolled metallic glass, Appl. Phys. Lett. 29 (1976) 328-330. 
[173] J. Pan, Q. Chen, L. Liu, Y. Li, Softening and dilatation in a single shear band, Acta Mater. 59 (13) (2011) 5146-5158. doi:10.1016/j.actamat. 2011.04 .047

[174] R. Maass, K. Samwer, W. Arnold, C. A. Volkert, A single shear band in a metallic glass: Local core and wide soft zone, Applied Physics Letters 105 (17) (2014) 171902. doi:10.1063/1.4900791.

[175] H. Shao, Y. Xu, B. Shi, C. Yu, H. Hahn, H. Gleiter, J. Li, High density of shear bands and enhanced free volume induced in $\mathrm{Zr}_{70} \mathrm{Cu}_{20} \mathrm{Ni}_{10}$ metallic glass by high-energy ball milling, Journal of Alloys and Compounds 548 (2013) 77-81. doi:10.1016/j.jallcom.2012.08.132.

[176] K.-W. Park, C.-M. Lee, M.-R. Lee, E. Fleury, M. L. Falk, J.-C. Lee, Paradoxical phenomena between the homogeneous and inhomogeneous deformations of metallic glasses, Applied Physics Letters 94 (2) (2009) 021907. doi:\{10.1063/1.3064920\}.

[177] R. D. Conner, W. L. Johnson, N. E. Paton, W. D. Nix, Shear bands and cracking of metallic glass plates in bending, Journal of Applied Physics 94 (2) (2003) 904-911. doi:10.1063/1.1582555.

[178] R. D. Conner, Y. Li, W. D. Nix, W. L. Johnson, Shear band spacing under bending of Zr-based metallic glass plates, Acta Materialia 52 (8) (2004) 2429-2434.

[179] L. H. Dai, Y. L. Bai, Basic mechanical behaviors and mechanics of shear banding in BMGs, International Journal of Impact Engineering 35 (8) (2008) 704-716. doi:10.1016/j.ijimpeng.2007.10.007.

[180] Y. F. Shi, M. L. Falk, Shear banding and short-range order in simulated nanoindentation of a metallic glass, in: P. Gumbsch (Ed.), Proceedings of the Third International Conference on Multiscale Materials Modeling, Fraunhofer IRB-Verl., Stuttgart, 2006, pp. 296-301. 
[181] V. Schmidt, H. Rösner, M. Peterlechner, G. Wilde, P. M. Voyles, Quantitative measurement of density in a shear band of metallic glass monitored along its propagation direction, Physical Review Letters 115 (2015) 035501.

[182] O. Haruyama, K. Kisara, A. Yamashita, K. Kogure, Y. Yokoyama, K. Sugiyama, Characterization of free volume in cold-rolled $\mathrm{Zr}_{55} \mathrm{Cu}_{30} \mathrm{Ni}_{5} \mathrm{Al}_{10}$ bulk metallic glasses, Acta Materialia 61 (9) (2013) 3224-3232. doi:10.1016/j.actamat.2013.02.010.

[183] M. Stolpe, J. J. Kruzic, R. Busch, Evolution of shear bands, free volume and hardness during cold rolling of a Zr-based bulk metallic glass, Acta Materialia 64 (2014) 231-240. doi:10.1016/j.actamat.2013.10.035.

[184] J. Bokeloh, S. V. Divinski, G. Reglitz, G. Wilde, Tracer measurements of atomic diffusion inside shear bands of a bulk metallic glass (vol 107,

口 235503, 2011), Physical Review Letters 107 (26) (2011) 235503. doi: 10.1103/PhysRevLett.107.269901.

[185] G. Wilde, H. Roesner, Nanocrystallization in a shear band: An in situ

a investigation, Applied Physics Letters 98 (2011) 251904. doi:10.1063/ 1.3602315 .

[186] Y. Shao, K. Yao, M. Li, X. Liu, Two-zone heterogeneous structure within shear bands of a bulk metallic glass, Applied Physics Letters 103 (17) (2013) 171901.

[187] R. Maaß, D. Klaumünzer, E. I. Preiß, P. M. Derlet, J. F. Löffler, Single shear-band plasticity in a bulk metallic glass at cryogenic temperatures, Scripta Materialia 66 (5) (2012) 231-234.

[188] H. Kimura, T. Masumoto, A model of the mechanics of serrated flow in an amorphous alloy, Acta Metallurgica 31 (2) (1983) 231-240. doi: $\{10.1016 / 0001-6160(83) 90100-1\}$. 
[189] J. Antonaglia, W. J. Wright, X. Gu, R. R. Byer, T. C. Hufnagel, M. LeBlanc, J. T. Uhl, K. A. Dahmen, Bulk metallic glasses deform via slip avalanches, Physical Review Letters 112 (15) (2014) 155501. doi:\{10.1103/PhysRevLett.112.155501\}.

[190] P. Rodriguez, Serrated plastic flow, Bulletin of Materials Science 6 (4) (1984) 653-663.

[191] J. M. Robinson, M. P. Shaw, Microstructural and mechanical influences on dynamic strain-aging phenomena, International Materials Reviews 39 (3) (1994) 113-122.

[192] R. Maaß, D. Klaumünzer, J. F. Löffler, Propagation dynamics of individual shear bands during inhomogeneous flow in a Zr-based bulk metallic glass, Acta Materialia 59 (8) (2011) 3205-3213.

[193] E. K. H. Salje, K. A. Dahmen, Crackling noise in disordered materials, Annual Review of Condensed Matter Physics 5 (1) (2014) 233-254.

[194] K. A. Dahmen, Y. Ben-Zion, J. T. Uhl, A simple analytic theory for the statistics of avalanches in sheared granular materials, Nature Physics 7 (7) (2011) 554-557.

[195] K. Dahmen, Y. Ben-Zion, J. Uhl, Micromechanical model for deformation in solids with universal predictions for stress-strain curves and slip avalanches, Physical Review Letters 102 (17) (2009) 175501.

[196] J. Antonaglia, X. Xie, G. Schwarz, M. Wraith, J. Qiao, Y. Zhang, P. K. Liaw, J. T. Uhl, K. A. Dahmen, Tuned critical avalanche scaling in bulk metallic glasses, Scientific Reports 4 (2014) 4382. doi: $10.1038 /$ srep04382

[197] K. Wang, T. Fujita, Y. Q. Zeng, N. Nishiyama, A. Inoue, M. W. Chen, Micromechanisms of serrated flow in a $\mathrm{Ni}_{50} \mathrm{Pd}_{30} \mathrm{P}_{20}$ bulk metallic glass with a large compression plasticity, Acta Mater. 56 (12) (2008) 28342842. doi:10.1016/j.actamat.2008.02.015 
[198] L. Tian, Y.-Q. Cheng, Z.-W. Shan, J. Li, C.-C. Wang, X.-D. Han, J. Sun, E. Ma, Approaching the ideal elastic limit of metallic glasses, Nat. Commun. 3 (2012) 609. doi:10.1038/ncomms1619.

[199] S. Takayama, Drawing of $\mathrm{Pd}_{77.5} \mathrm{Cu}_{6} \mathrm{Si}_{16.5}$ metallic glass wires, Materials

[204] W. F. Wu, Y. Li, C. A. Schuh, Strength, plasticity and brittleness of bulk metallic glasses under compression: statistical and geometric effects, Philos. Mag. 88 (1) (2008) 71-89. doi:10.1080/14786430701762619.

[205] C. C. Hays, C. P. Kim, W. L. Johnson, Microstructure controlled shear 1710 
[206] C. Fan, R. T. Ott, T. C. Hufnagel, Metallic glass matrix composite with precipitated ductile reinforcement, Appl. Phys. Lett. 81 (2002) 1020.

[208] Q. Zheng, J. Du, Low beryllium content Zr-based bulk metallic glass com-

[207] M. L. Lee, Y. Li, C. A. Schuh, Effect of a controlled volume fraction of dendritic phases on tensile and compressive ductility in La-based metallic glass, Acta Mater. 52 (14) (2004) 4121-4131. doi:10.1016/j.actamat. 2004.05 .025 . posite with plasticity and work hardenability, J. Appl. Phys. 115 (2014) 043519. doi:10.1063/1.4863454.

[209] J. Das, M. B. Tang, K. B. Kim, R. Theissmann, F. Baier, W. H. Wang, J. Eckert, "Work-hardenable" ductile bulk metallic glass, Phys. Rev. Lett. 94 (20) (2005) 205501. doi:10.1103/PhysRevLett.94.205501.

[210] S. W. Lee, M. Y. Huh, E. Fleury, J. C. Lee, Crystallization-induced plasticity of $\mathrm{Cu}-\mathrm{Zr}$ containing bulk amorphous alloys, Acta Mater. 54 (2) (2006) 349-355. doi:10.1016/j . actamat.2005.09.007

[211] J. Fornell, E. Rossinyol, S. Surinach, M. D. Baro, W. H. Li, J. Sort, Enhanced mechanical properties in a Zr-based metallic glass caused by deformation-induced nanocrystallization, Scr. Mater. 62 (1) (2010) 13-16. doi:10.1016/j.scriptamat.2009.09.014.

[212] A. Inoue, W. Zhang, T. Tsurui, A. R. Yavari, A. L. Greer, Unusual roomtemperature compressive plasticity in nanocrystal-toughened bulk copper-

1 zirconium glass, Philos. Mag. Lett. 85 (5) (2005) 221-229. doi:10.1080/ 09500830500197724

[213] S. Pauly, S. Gorantla, G. Wang, U. Kuehn, J. Eckert, Transformationmediated ductility in CuZr-based bulk metallic glasses, Nat. Mater. 9 (6) (2010) 473-477. doi:10.1038/NMAT2767.

[214] Q. P. Cao, J. W. Liu, K. J. Yang, F. Xu, Z. Q. Yao, A. Minkow, H. J. Fecht, J. Ivanisenko, L. Y. Chen, X. D. Wang, S. X. Qu, J. Z. Jiang, Effect 
of pre-existing shear bands on the tensile mechanical properties of a bulk metallic glass, Acta Mater. 58 (4) (2010) 1276-1292. doi:10.1016/j. actamat.2009.10.032.

[215] W. H. Jiang, F. Jiang, F. X. Liu, Y. D. Wang, H. M. Dang, F. Q. Yang, H. Choo, P. K. Liaw, Mechanical behaviours of workhardening and worksoftening bulk metallic glasses, Mater. Sci. Technol. 28 (2) (2012) 249-255. doi:10.1179/1743284711Y.0000000011

[216] S. Takayama, Work-hardening and susceptibility to plastic-flow in metallic glasses (rolling deformation), J. Mater. Sci. 16 (9) (1981) 2411-2418.

[217] K. B. Kim, J. Das, S. Venkataraman, S. Yi, J. Eckert, Work hardening ability of ductile $\mathrm{Ti}_{45} \mathrm{Cu}_{40} \mathrm{Ni}_{7.5} \mathrm{Zr}_{5} \mathrm{Sn}_{2.5}$ and $\mathrm{Cu}_{47.5} \mathrm{Zr}_{47.5} \mathrm{Al}_{5}$ bulk metallic glasses, Appl. Phys. Lett. 89 (7) (2006) 071908. doi:10.1063/1.2337534.

[218] K. F. Yao, F. Ruan, Y. Q. Yang, N. Chen, Superductile bulk metallic glass, Appl. Phys. Lett. 88 (12) (2006) 122106. doi:10.1063/1.2187516

[219] S.-C. Lee, M.-Y. Huh, H.-J. Kim, J.-C. Lee, Extraordinary plasticity of an amorphous alloy based on atomistic-scale phase separation, Mater. Sci. Eng. A-Struct. Mater. Prop. Microstruct. Process. 485 (1-2) (2008) 61-65. doi:10.1016/j.msea.2007.08.068

[220] A. I. Taub, F. Spaepen, The kinetics of structural relaxation of a metallic glass, Acta Metall. 28 (1980) 1781.

[221] M. Tao, A. H. Chokshi, R. D. Conner, G. Ravichandran, W. L. Johnson, Deformation and crystallization of Zr-based amorphous alloys in homogeneous flow regime, Journal of Materials Research 25 (06) (2011) 11371148 .

[222] Z. T. Wang, J. Pan, Y. Li, C. A. Schuh, Densification and strain hardening of a metallic glass under tension at room temperature, Phys. Rev. Lett. 111 (2013) 135504. doi:10.1103/PhysRevLett.111.135504. 
[223] C. E. Packard, L. M. Witmer, C. A. Schuh, Hardening of a metallic glass during cyclic loading in the elastic range, Appl. Phys. Lett. 92 (2008)

[224] C. Deng, C. A. Schuh, Atomistic mechanisms of cyclic hardening in metal-

प

[225] C. E. Packard, E. R. Homer, N. Al-Aqeeli, C. A. Schuh, Cyclic harden1775

I

[226] E. Cubuk, S. S. Schoenholz, J. M. Rieser, B. D. Malone, J. Rottler, E. Durian, E. Kaxiras, A. J. Liu, Fracture toughness of metallic glasses:

[227] A. Hirata, L. J. Kang, T. Fujita, B. Klumov, K. Matsue, M. Kotani, A. R. Yavari, M. W. Chen, Geometric frustration of icosahedron in metallic glasses, Science 341 (6144) (2013) 376-379.

[228] A. Hirata, P. Guan, T. Fujita, Y. Hirotsu, A. Inoue, A. R. Yavari, T. Saku- 
